\title{
Dependencias no locales y cadenas de verbos auxiliares ${ }^{1}$
}

\author{
Non-local dependencies and auxiliary chains
}

\author{
Luis García Fernández \& Diego Gabriel Krivochen \\ Universidad Complutense de Madrid - University of Reading
}

—Ascolta! Più tempo non ho.

-Parla, parla, ascoltando ti sto.

W. A. Mozart - L. Da POnTE, Don Giovanni, escena 6

\begin{abstract}
ReSumen. El objetivo de este trabajo es entender el comportamiento de las perífrasis verbales y, especialmente, de las perífrasis verbales formadas por más de un auxiliar, es decir, aquellas en las que hay una cadena de auxiliares. Para ello, se aportarán datos que afectan a dos fenómenos de desplazamiento de constituyentes: por un lado, la anteposición de la forma no finita del verbo léxico (específicamente, gerundios) y el consecuente problema de la posición del sujeto y, por otro, la subida de clíticos. Mostraremos que estos casos de anteposición de gerundio presentan dificultades teóricas y empíricas para los modelos de estructura de frase ortodoxos, que pueden no obstante solucionarse adoptando un enfoque de desplazamiento como multidominancia en una gramática de adjunción lexicalizada. La solución propuesta aquí, argumentaremos, puede extenderse a otras áreas de la gramática, como el movimiento Qu-.
\end{abstract}

Palabras clave: anteposición, clíticos, perífrasis verbales, verum focus.

Data de recepción: 26-01-2018 - Data de aceptación: 10-05-2018.

1 Este trabajo ha sido parcialmente financiado por el Ministerio de Economía y Competitividad dentro del proyecto Cadenas de verbos auxiliares en español (ref.: FFI2015-68656-P). 
ABSTRACT. The goal of the present paper is to explain the behaviour of verbal periphrases and, particularly, those composed by more than a single auxiliary verb, i.e. those cases in which there is an auxiliary chain. To this end, we will provide data pertaining to two displacement phenomena: first fronting of a non-finite lexical verb (gerunds, specifically) and the concomitant problem of the surface position of the subject, and secondly, clitic climbing. We will show that these instances of fronting pose both theoretical and empirical problems for orthodox approaches to phrase structure, which can be solved by adopting a view of displacement as multidominance within a lexicalized Tree Adjoining Grammar. We will also argue that this solution can be extended to other areas of the grammar such as Wh-movement.

Keywords: fronting, clitics, verbal periphrases, verum focus.

\section{INTRODUCCIÓN}

\subsection{El concepto de verum focus}

El verum focus es, semánticamente, un foco centrado en la polaridad de una oración declarativa que introduce un conjunto de alternativas que contrastan con el significado no marcado de la oración (Escandell Vidal \& Leonetti 2009: 15-16). En este trabajo analizaremos una serie de casos que ejemplifican una de las formas en las que el español marca el verum focus, a saber, la anteposición no escindida. Este es un tipo de anteposición que induce un foco de polaridad, distinta del foco contrastivo y de otras anteposiciones identificadas en la bibliografía (Leonetti \& Escandell Vidal 2009; Escandell Vidal \& Leonetti 2009; Jiménez Fernández 2015, en prensa). A los fines del presente trabajo, en la medida en que nos ocuparemos de describir los mecanismos sintácticos mediante los cuales se produce la anteposición de gerundios modificados por una cadena de verbos auxiliares, utilizaremos verum focus como forma abreviada para anteposición inductora de verum focus o anteposición inductora de foco de polaridad.

Conviene empezar distinguiendo la construcción que nos ocupa de otros tipos de anteposición focal discutidas en la literatura y describiendo las propiedades adscritas al verum focus. Así, en (1) tenemos un ejemplo de foco contrastivo (las mayúsculas indican prominencia fonológica), mientas que en (2) tenemos uno de verum focus:

(1) EL LIBRO ya he terminado (no los artículos)

(2) Nada tengo que añadir a lo que ya dije en su día (anteposición de nada) (Los ejemplos de (1) y (2) son de Leonetti \& Escandell Vidal 2009: 156) 
Este tipo de anteposición se distingue sistemáticamente de focos contrastivos, focos mirativos (mirative focus) y dislocación a la izquierda (clitic left dislocation, ver particularmente Leonetti \& Escandell Vidal 2009; también Jiménez Fernández 2015 para una tipología de focos y otras anteposiciones no interrogativas). Las principales características del verum focus, tal y como aparecen descritas en Leonetti y Escandell Vidal (2009: 159-165) (ver también Hernanz \& Brucart 1987), son las siguientes:

- No hay entonación enfática (en mayúsculas en (3a)), ni una pausa entre el elemento focalizado y el resto de la oración (en (3b), cfr. (1)):

(3) a. \#DE POCO te va a servir llamarle.

b. \#NADA, tengo que añadir.

- No se admiten clíticos reasuntivos:

(4) $* \mathrm{Poco}_{i}$ te $\mathrm{lo}_{i}$ puedo decir (adaptado de Leonetti \& Escandell Vidal 2009: 159)

Esta propiedad distingue al verum focus de las dislocaciones con clítico, sean a posiciones de tópico o foco.

- Leonetti y Escandell Vidal (2009: 159) señalan que se requiere adyacencia entre el elemento antepuesto y el verbo ${ }^{2}$, afirmación sobre la que volveremos en detalle, ya que resulta inadecuada en los casos en los que tenemos una cadena de verbos auxiliares modificando al verbo léxico:

(5) *Algo ella debe saber (tomado de Leonetti \& Escandell Vidal 2009: 160)

Esta última propiedad, que según Campos y Zampini (1990) y Hernanz y Brucart (1987) no es obligatoria en el caso del foco contrastivo, resulta particularmente relevante a los propósitos de este trabajo, ya que nos permite analizar el comportamiento sintáctico del verum focus en comparación con las estructuras interrogativas. En efecto, el verum focus comparte algunas propiedades del movimiento de SQu-, entre ellas,

2 «[En los ejemplos de anteposición con verum focus] there is an adjacency requirement between the fronted constituent and the verb [lo que distingue al verum focus de la dislocación a la izquierda]» (Leonetti \& Escandell 2009: 159; destacado nuestro); del mismo modo, Escandell y Leonetti (2009: 24) afirman, en su resumen de las características de la anteposición inductora de foco de polaridad, que «El sujeto aparece obligatoriamente en posición posverbal (debido al requisito de adyacencia entre el verbo finito y el constituyente antepuesto, como en las interrogativas parciales y en la focalización contrastiva)» (destacado nuestro). 
muy notablemente, la inversión sujeto-verbo. Desde este punto de vista, la agramaticalidad de (5) sería explicada por los mismos motivos que la agramaticalidad de (6):

(6) ¿Cómo Juan está?

Leonetti y Escandell Vidal (2009) y Escandell Vidal y Leonetti (2009) tratan casi exclusivamente de casos de verum focus en los que hay anteposición de un SN. Lo que nos interesa aquí, sin embargo, son aquellos casos en que lo que se antepone es el verbo léxico que sigue a un verbo auxiliar, como sucede en los dos ejemplos de (7):

(7) a. Escuchándote estoy.

b. Muriéndome tendría que estar yo para no ir a la fiesta.

En (7b), tenemos más de un auxiliar, lo que en RAE-ASALE (2009: §28.1) y Bravo et alii (2015) se denomina una «cadena de verbos auxiliares». En este trabajo argumentaremos que la anteposición de los gerundios en (7) ha de estudiarse como un caso de anteposición inductora de foco de polaridad (o simplemente verum focus, en línea con lo dicho más arriba): estamos frente a una anteposición no contrastiva ni tópica que cumple con las condiciones para ser considerada parte del fenómeno de verum focus. Veamos algunas de las propiedades comunes entre la construcción con gerundio que nos interesa y los casos analizados por Leonetti y Escandell Vidal:

(8) Incompatibilidad con la aparición de clíticos reasuntivos:

a. *Muriéndome tendría que estarlo yo para no ir a la fiesta.

Ausencia de entonación enfática:

b. \#? ESCUCHÁNDOTE estoy.

Posibilidad de parafrasear la construcción antepuesta con una estructura escindida con una partícula polar positiva (cfr. Escandell \& Leonetti 2009: §3.2):

c. -Escúchame!

—Escuchándote estoy $\sim$ sí que estoy escuchándote.

En lo que queda del trabajo daremos cuenta de las posibles configuraciones que surgen cuando tenemos anteposición de un gerundio a la izquierda de una cadena de verbos auxiliares y dos cuestiones importantes relacionadas con este proceso: la posición del sujeto en la estructura derivada y la posición de los argumentos internos del verbo, particularmente clíticos. Nuestro foco estará puesto en las propiedades sintácticas de las construcciones, aunque esto no quiere decir que la anteposición del 
gerundio no tenga una motivación semántica o pragmática (relacionada con estructura informativa) y una manifestación sintáctica (antes que una motivación sintáctica, como puede serlo el cotejo de rasgos formales criteriales en el sentido de Rizzi 2010, que forman parte de las motivaciones intra-teóricas del Programa Minimalista). A tal efecto, haremos uso de las herramientas que nos proporcionan modelos de estructura de frase como la Gramática de Adjunción de Arboles (Tree Adjoining Grammars; Joshi 1985; Joshi \& Kroch 1985) en su variante lexicalizada (Frank 1992, 2002) y los modelos de desplazamiento como Multidominancia en lugar de movimiento literal de constituyentes (Citko 2014; McCawley 1982, 1998). Veremos que hay razones no solamente teóricas (relacionadas con el poder generativo fuerte de cada teoría, el grado de restricciones que presentan y la necesidad o no de adoptar supuestos innecesariamente ad hoc) sino fundamentalmente empíricas (relacionadas con la adecuación de las descripciones estructurales que generamos a los datos lingüísticos presentados) para la elección de estas herramientas.

\subsection{Verum focus y cadenas de verbos auxiliares: los datos}

La anteposición del verbo léxico de una perífrasis verbal plantea dos cuestiones básicas de orden de palabras. La primera afecta a la posición del sujeto y, la segunda, a la posición de los pronombres clíticos que han experimentado subida. Vamos a exponer muy brevemente estas dos cuestiones.

Como acabamos de señalar, Leonettti y Escandell Vidal (2009: 160) observan que el verum focus comparte con el movimiento Qu- la inversión del orden sujeto-verbo, como se muestra en (9) y (10):

(9) a. *Nada yo tengo que decir.

b. Nada tengo yo que decir.

(10) a. ¿¿Qué tú tienes que decir?

b. ¿Qué tienes tú que decir?

La inversión del orden sujeto-verbo en el movimiento Qu- ha sido muy estudiada (Emonds 1970; Chomsky 1977; Torrego 1984, entre otros muchos). Lo que nos interesa aquí es un problema empírico que surge con las perífrasis verbales en las que hay más de un auxiliar y es que el sujeto no puede estar delante del verbo finito (11a), pero no tiene que estar necesariamente detrás del último auxiliar (11b), sino que puede ocupar posiciones intermedias (11c). Es decir, se puede determinar que la posición del sujeto 
no será a la izquierda del verbo finito, pero no se puede determinar a la derecha de qué verbo se manifestará:

(11) a. *Muriéndome yo tendría que estar para no ir a la fiesta.

b. Muriéndome tendría que estar yo para no ir a esa fiesta.

c. Muriéndome tendría yo que estar para no ir a esa fiesta.

Es necesario, pues, establecer de qué manera se puede cumplir la inversión del sujeto a la que obliga el verum focus (y el movimiento Qu-) en los casos en que hay una cadena de verbos auxiliares.

La segunda cuestión objeto de nuestro trabajo es, como hemos señalado, la interacción del verum focus con la subida de clíticos. En el caso de que el verum focus, en tanto operación, afecte al verbo léxico de una perífrasis del cual dependa un objeto pronominal, tenemos dos posibles posiciones lógicas para el clítico antepuesto: enclítico al auxiliado o proclítico al auxiliar. Las segmentaciones posibles para una secuencia como (12) son bien (13) o bien (14) (donde + ha de interpretarse como concatenación lineal):

(12) a. Gerundio + clítico + auxiliar

b. Escuchando + te + estoy.

(13) a. [Gerundio + clítico] + auxiliar

b. [Escuchándote] estoy.

(14) a. Gerundio $+[$ clítico + auxiliar $]$

b. Escuchando [te estoy].

La cuestión que se nos presenta es que la segmentación de (14) es agramatical, mientras que la de (13) es gramatical. Trataremos de explicar por qué mediante el aparato teórico que presentaremos en el apartado 2 y volveremos sobre esta cuestión en el apartado 4.

En las secciones siguientes presentaremos una hipótesis respecto de la derivación de la anteposición de gerundio con verum focus que, por un lado, intenta capturar las restricciones sobre la posición de los sujetos y, por el otro, hacer explícito cómo interactúa la anteposición con la subida de clíticos. Veremos que podemos dar cuenta de ambos fenómenos de manera unificada con el modelo que desarrollamos en este trabajo y que hemos presentado en trabajos anteriores. 


\section{APARATO TEÓRICO}

El aparato teórico que usaremos aquí requiere la introducción de algunas nociones que hemos presentado en trabajos anteriores sobre las propiedades sintáctico-semánticas de los verbos auxiliares y las cadenas que forman. En Krivochen y García Fernández (en prensa) desarrollamos la idea expuesta en Bravo et alii (2015) de que la categoría de verbos auxiliares no es homogénea ni sintáctica ni semánticamente. Consideremos inicialmente los siguientes ejemplos:

(15) a. Juan puede estar trabajando.

b. Juan está pudiendo trabajar.

Resulta evidente que (15a) y (15b) no significan lo mismo; menos evidente resulta, no obstante, señalar que por este hecho deben recibir segmentaciones diferentes. Es necesario notar que cualquier gramática de estructura de frase (basada en Satisfacer, como GB, o en Ensamble, como el PM) únicamente pueden generar la siguiente segmentación, correspondiente a una estructura estrictamente monotónica ${ }^{3}$ (siempre extendiendo el marcador de frase en la raíz de manera binaria, la llamada condición de extensión en Chomsky 1995):

(16) a. [Juan [puede [estar [trabajando]]]

b. [Juan [está [pudiendo [trabajar]]]

Las etiquetas particulares que se usen para cada proyección no son pertinentes ahora; sí lo es el hecho de que, independientemente de cómo llamemos a cada proyección sintagmática, las segmentaciones de (16) no son capaces de dar cuenta adecuadamente de las diferencias de significado, que simplemente no pueden capturarse en un modelo en el cual la representación semántica se construye después de la sintáctica (y la sintáctica se basa en un esqueleto a priori determinado por la Gramática Universal, como en Cinque 1999).

Matemáticamente, una función es monotónica (o monótona) si se comporta de manera uniforme en todo su dominio (es decir, si crece o decrece siempre, y siempre al mismo ritmo, la derivada de la función debe ser constante). El sistema derivacional propuesto en Chomsky (1995 y trabajos posteriores), en el cual la derivación procede paso a paso manipulando objetos $\{\mathrm{X}, \mathrm{SY}\}$, es efectivamente monotónico debido a las exigencias de linealización impuestas por el Axioma de Correspondencia Lineal (Kayne 1994). Nuestro argumento a favor de una estructura de frase no monotónica es esencialmente empírico, aunque puede verse Uriagereka (2002) para un argumento formal respecto de la naturaleza no monotónica de ciertos objetos sintácticos a nivel local. 
Hemos dicho que (15a) y (15b) no son sinónimas. Obsérvese que ello solo puede explicarse si en (15b) el auxiliar de Progresivo no auxilia al verbo trabajar. En efecto, en (15b) es necesario que tener que auxilie a trabajar puesto que es el auxiliar inmediatamente adyacente. En estas circunstancias, si estar auxiliase a trabajar como hace en (15a), las dos secuencias tendrían que ser necesariamente sinónimas, pero no lo son. En (15a) se afirma la necesidad, epistémica o deóntica, de que se dé la situación de que Juan esté trabajando, mientras que en (15b) se afirma que actualmente existe para Juan la obligación deóntica de trabajar, pero no que en el momento del habla lo esté haciendo. Creemos que la diferencia semántica entre ambas oraciones es clara e indiscutible; resulta lógico, luego de identificarla, intentar representar esa diferencia semántica en la segmentación. De este modo, es necesario que la sintaxis pueda generar las dos descripciones estructurales en (17):

(17) a. [Juan [puede [estar trabajando]]]

b. [Juan [está pudiendo [trabajar]]]

Resumiendo lo sostenido en Bravo et alii (2015), García Fernández et alii (2017) y Krivochen y García Fernández (en prensa), una sintaxis adecuada ${ }^{4}$ (en el sentido de Joshi 1985: 208) para las cadenas de verbos auxiliares en español necesita ser capaz de generar tanto (17a) como (17b). Crucialmente, esto no es posible en un modelo monotónico: ciertamente la estructura crece en cada paso derivacional, pero no al mismo ritmo: en (b) el objeto sintáctico [está pudiendo] tiene que modificar al $\mathrm{SV}$, encabezado por trabajar. Es importante también que estar no modifica al verbo léxico, sino solamente al auxiliar poder; en términos formales, estar no tiene alcance (scope) sobre trabajar. Nótese que si el alcance de X se define como el conjunto de nodos que X manda-c en Forma Lógica (Ladusaw 1980; May 1985, entre muchos otros) entonces no puede ser el caso que estar mande-c a trabajar. En los trabajos mencionados propusimos que las cadenas de verbos auxiliares en español no tienen una estructura monotónica (ver Schmerling 1983 para algunas conclusiones similares

«A grammar $G$ is weakly adequate for a string language $L$ if $L(G)=L$. G is strongly adequate for $\mathrm{L}$ if $\mathrm{L}(\mathrm{G})=\mathrm{L}$ and for each $\mathrm{w}$ [a string in $\mathrm{L}$ ] in $\mathrm{L}, \mathrm{G}$ assigns an 'appropriate' structural description to w» (Joshi 1985: 208).

El concepto de «descripción estructural apropiada» implica evitar asignar estructura extra en la forma de nodos terminales o no terminales que no tengan motivación independiente. En Krivochen (2015) argumentamos que este requerimiento sugiere un modelo de estructura de frase computacionalmente mixta, que combina sub-estructuras Markovianas con sub-estructuras libres de contexto y hasta débilmente sensibles al contexto. 
respecto de las cadenas inglesas), sino que deben segmentarse en dominios locales. En estos trabajos distinguimos dos clases de verbos auxiliares:

(I) AUXILIARES LÉXicos, que, a pesar de ser auxiliares, tienen un significado próximo a lo que se considera habitualmente como significado léxico en tanto pueden ser argumentos de otros auxiliares, los del segundo tipo, a la vez que predicados de otros auxiliares léxicos o de verbos léxicos plenos. A esta categoría pertenecen los auxiliares modales (epistémicos y deónticos) y aspectuales fasales.

(II) AUXILIARES FUNCIONALES, que aportan información temporal y aspectual y modifican a verbos léxicos o a auxiliares léxicos, pero no son ellos mismos objeto de modificación.

El siguiente cuadro resume el estado de cosas con algunos ejemplos:

\begin{tabular}{|c|c|}
\hline Auxiliares funcionales & Auxiliares léxicos \\
\hline $\begin{array}{l}\text { Estar progresivo, ser de pasiva, haber } \\
\text { perfectivo, ir a de futuro. }\end{array}$ & $\begin{array}{l}\text { Fasales (empezar a; terminar de...), } \\
\text { modales (epistémicos / deónticos), } \\
\text { auxiliares de primera posición (soler, } \\
\text { haber de), tardar en. }\end{array}$ \\
\hline
\end{tabular}

La noción de modificación que asumimos es la estándar: en $a(b), a$ modifica a $b$ (independientemente de si $a$ selecciona o subcategoriza a $b$ : la relación vale tanto para predicados-argumentos como para predicados-adjuntos).

La ampliación de esta idea en Krivochen y García Fernández (en prensa) introduce la noción de «proyección extendida» (PE) de un predicado léxico (basada en Grimshaw 2005 y Abney 1987): esta contiene al predicado léxico (sea un verbo o un auxiliar) y a sus dependientes (auxiliares funcionales y argumentos). Veamos un ejemplo, con la segmentación en PPEE marcada con paréntesis cuadrados y los auxiliares léxicos en cursiva:

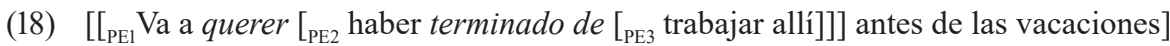

PE1 y PE2 tienen sendos auxiliares léxicos con sus correspondientes modificadores funcionales. La proyección extendida de querer (PE1) modifica a la proyección extendida del fasal terminar de (PE2) y esta modifica al SV encabezado por trabajar (PE3).

En el presente trabajo, refinamos esa idea: la PE de un predicado incluye no solo a sus dependientes funcionales (es decir, a los auxiliares funcionales que lo modifiquen), 
sino también a los argumentos que licencie: sujetos y objetos. Inicialmente, esto significa que hay una ocurrencia de cada dependiente en cada PE, ya que en principio los dependientes pueden aparecer en posiciones internas en una cadena de auxiliares. Esto podría formalizarse, por ejemplo, mediante movimiento cíclico de los dependientes desde una posición-A en el SV a posiciones dentro de cada PE. Una derivación en términos de movimiento de PE en PE sería como en (19):

(19) a. Juan puede tener que empezar a escribir el libro.

b. Juan ${ }_{i} l_{j}$ puede tener $h_{i} h_{j}$ que empezar $h_{i} h_{j}$ a $h_{i}$ escribir $h_{j}$

No obstante, hay restricciones respecto de la realización de estos dependientes nominales dentro de la PE de un predicado en una cadena de auxiliares: por un lado, estos dependientes no pueden ser sintagmas fonológicamente pesados ${ }^{5}$; por el otro, no pueden aparecer relacionados con otros dependientes del núcleo léxico. Es decir, si la PE de un auxiliar léxico comprende a este auxiliar, más un auxiliar funcional (como en «haber $r_{\mathrm{FUNC}}$ tenido $\left.\mathrm{que}_{\mathrm{LEX}} \ldots \gg\right)$, el sujeto no puede depender del auxiliar funcional, sino solo del léxico, ya que los dependientes nominales están licenciados por el núcleo de las PPEE$^{6}$. Ambas restricciones son incompatibles con una visión estrictamente

5 Cabe destacar que esto es una preferencia antes que una restricción. Los sintagmas fonológicamente pesados tienden a aparecer en las periferias oracionales (fundamentalmente, la derecha) antes que en posiciones internas, lo cual en general se atribuye a reglas estilísticas antes que sintácticas. Ejemplos de este tipo de desplazamiento por razones prosódicas son el llamado Heavy NP shift y la extraposición de cláusula relativa, ejemplificados en (i) y (ii) respectivamente:

i) I sent $h_{i}$ to Mary $\left[_{\mathrm{SN}} \text { that book about grammar that she liked so very much }\right]_{i}\left(c f r\right.$. ${ }^{*}$ I sent to Mary it)

i) There came a man $h_{i}$ to the store [who was smoking Cuban cigars]

6 A este respecto Torrego (1984: 105) observa que en casos en los que hay más de un verbo (es decir, en construcciones perifrásticas), «only the first verb of the sequence must necessarily precede the subject». No obstante, observa que esta generalización se aplica a los modales y aspectuales de fase, aunque «an exception to this generalization is the behaviour of the auxiliaries ser and haber»" en el caso de las interrogativas $\mathrm{Qu}-$, ya que no pueden preceder inmediatamente al sujeto en casos de inversión (los ejemplos y los juicios son de Torrego):

i) $*_{\text {¿Qué ha la gente organizado? }}$

ii) * ¿Por quién fue la reunión organizada?

Torrego no se pregunta, no obstante, qué ocurre cuando estamos en presencia de una cadena de auxiliares (es decir, más de un auxiliar frente al verbo principal). Parece haber diferencias entre las interrogativas Qu- y las interrogativas polares respecto de la posibilidad de admitir ser inmediatamente antes del sujeto, en vista de ejemplos como:

iii) ¿Será Juan condenado por ese delito?

No obstante, en este trabajo nos ocupamos de cadenas en las que se genera una relación Operador-variable, que no es el caso en las interrogativas polares. Los datos en las interrogativas polares 
monotónica de la estructura sintáctica en la que el movimiento de constituyentes sea irrestricto (es decir, no regido léxicamente).

\section{LA POSICIÓN DEL SUJETO}

En esta sección vamos a ocuparnos del primer grupo de datos empíricos al que hemos hecho alusión en la sección 1.2, la posición del sujeto en una cadena de verbos auxiliares con una anteposición del verbo léxico por verum focus. Comencemos por proponer una oración con una cadena de verbos auxiliares (20a) a la que vamos a aplicar el movimiento de verum focus al verbo léxico, en este caso, un gerundio (20b).

(20) a. Tendría que estar muriéndome para no ir a esa fiesta.

b. Muriéndome tendría que estar para no ir a esa fiesta.

Como ya hemos visto a propósito de estos ejemplos en (11), la cuestión es que en la estructura de (20b), con el gerundio antepuesto por verum focus, el sujeto no puede estar delante del verbo finito tendría (21a), pero sí puede estar detrás de él (21b) o detrás del segundo auxiliar, estar (21c) ${ }^{7}$ :

(21) a. *Muriéndome yo tendría que estar para no ir a la fiesta (cfr. (6))

b. Muriéndome tendría yo que estar para no ir a esa fiesta.

c. Muriéndome tendría que estar yo para no ir a esa fiesta.

Si la posición canónica de los sujetos superficiales es el especificador del primer núcleo en la estructura que se asignaría a (21) y si solo se antepusiera el gerundio, (21a) debería ser gramatical, pero no lo es. Ello quiere decir que tiene que producirse

tampoco están del todo claros, ya que (iv), donde el auxiliar poder aparece en una forma compuesta con haber, resulta agramatical (ver también Zagona 2003: 167):

iv) ¿ ¿Habrá Juan podido entregar el trabajo a tiempo?

Más allá del interés innegable de estas construcciones, no profundizaremos en ellas en este trabajo.

7 En este trabajo vamos a usar únicamente ejemplos de verum focus, pero la posición del sujeto se plantea en los mismos términos si tenemos movimiento $\mathrm{Q}$ y cadenas de auxiliares:

(i) a. *AA quién Juan podría estar acosando?

b. ¿A quién podría Juan estar acosando?

c. ¿A quién podría estar Juan acosando?

d. ¿A quién podría estar acosando Juan?

Obsérvese que los ejemplos de (ia), (ib) e (ic) se corresponden, respectivamente, a los de (21a), (21b) y (21c). (id) es distinto porque el hecho de que el gerundio no se haya movido proporciona una nueva posición para el sujeto. 
un movimiento ulterior de la cadena de auxiliares, que explica la agramaticalidad de (21a). Lo que nos interesa subrayar ahora es que este movimiento no es necesariamente la subida del primer auxiliar (es decir, movimiento de Aux a C), lo que produciría únicamente el orden que observamos en (21b), sino que, como sucede en el movimiento Qu- en (7) (ver también (32), abajo), el sujeto no puede aparecer a la izquierda del verbo finito, pero tampoco ha de quedar necesariamente a la derecha de él, puesto que en (21c) aparece detrás de los dos auxiliares. Es decir, hay más de una manera sintáctica de satisfacer la necesidad de que el sujeto no esté inmediatamente a la derecha del elemento focalizado: (21b) es una manera y (21c) es otra8.

A continuación vamos a presentar las dos opciones lógicas para la anteposición de los auxiliares en los ejemplos de verum focus en los modelos tradicionales de estructura de frase (en Rección y Ligamiento y el Programa Minimalista), que asumen que la propiedad del desplazamiento ha de modelarse mediante transformaciones de movimiento:

(i) La cadena de auxiliares se antepone por movimiento de núcleo a núcleo, cada auxiliar por su cuenta.

(ii) La cadena de auxiliares se antepone como objeto complejo (movimiento de $\mathrm{SX}$, necesariamente a una posición de especificador).

Vamos a analizar ambas opciones en detalle, para mostrar que una solución transformacional en un modelo de estructura de frase monotónica (como X-barra o Ensamble binario) no da cuenta adecuadamente de los datos. En este punto, surge la siguiente cuestión: si los miembros de la cadena se anteponen individualmente, como núcleos (por ejemplo, a posiciones de Tópico o Foco; el problema que señalamos es más fundamental que las etiquetas de las proyecciones en la medida en que tiene que

8 Del mismo modo, la propuesta de que los sujetos en español pueden no subir al especificador de $\mathrm{T} / \mathrm{Flex}$, sino que pueden quedarse en el SV en ciertos contextos (fundamentalmente interrogativos, e.g., Uribe-Etxebarria 1992; Barbosa 2001) resulta igualmente insuficiente. La forma no finita del verbo sube a una posición por encima de T/Flex en la periferia izquierda, y hay varias posiciones para que se materialice el sujeto de ese verbo. Sostener que en casos de «inversión» el sujeto se queda en el Especificador de SV (o Sv) y el rasgo EPP de T/Flex se satisface mediante movimiento de $\mathrm{V}$ a T/Flex plantea el mismo problema en las cadenas que sostener que el verbo sube a $\mathrm{C}$ y que la posición canónica del sujeto es el especificador de T/Flex: no se puede dar cuenta de la posibilidad de que el sujeto se materialice en posiciones internas a la cadena de auxiliares (es decir: la única opción posible debería ser (21c)). La exposición que sigue vale tanto para las propuestas de que el sujeto aparece en el especificador de T/Flex como para aquellas que sostienen que en casos de inversión el sujeto se queda en una posición inferior, en SV. 
ver con el poder generativo fuerte del sistema), tenemos dos problemas: (i) o bien no hay posiciones estructurales para que se realicen los sujetos en (18), porque los núcleos se mueven a núcleos, (ii) o bien se predice que, si los auxiliares se anteponen mediante movimiento de núcleo a núcleo a posiciones funcionales completas por encima de ST cada uno con su especificador, cada auxiliar puede legitimar una posición para un sujeto interno a la cadena de auxiliares, incluyendo al auxiliar más alto (contra (21a) y (6)).

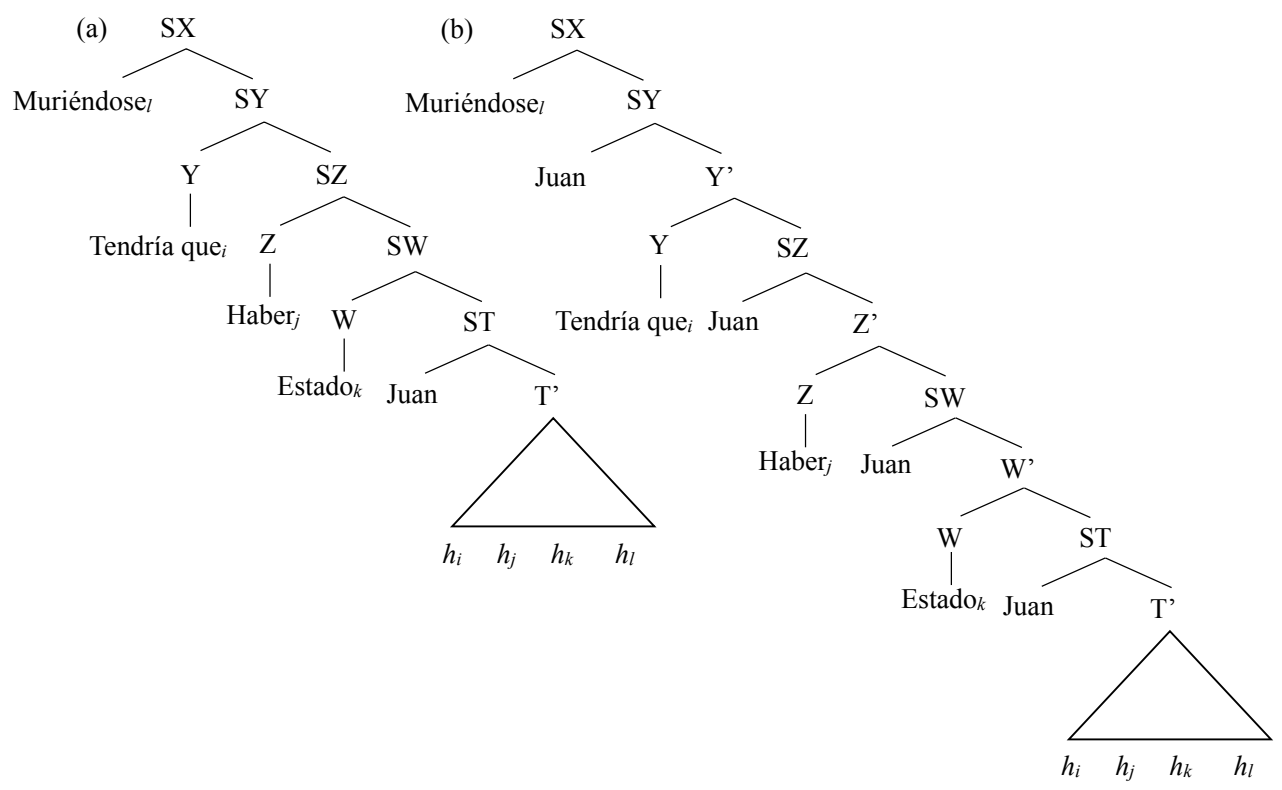

La representación de (22) derivada mediante movimiento de núcleos (en donde cada auxiliar en una cadena es un núcleo independiente y se mueve, en consecuencia, a un núcleo funcional diferente en la periferia izquierda; ver Emonds 2007 para la idea de la falta de determinación categorial en las «proyecciones discursivas»), resulta insuficiente por dos motivos alternativos: bien (i) porque no hay posiciones estructurales para realizar los sujetos internos a la cadena de auxiliares si las proyecciones a las que se mueven los auxiliares como núcleos no proyectan especificador (en un modelo de Estructura de Frase Escueta, como en (22a)), o bien (ii) porque, si cada proyección funcional sí legitima un especificador (como en (22b)), entonces debería ser posible tener un sujeto en todos y cada uno de los especificadores, incluyendo el más alto. Esto predice que debería ser posible tener al sujeto adyacente al gerundio 
antepuesto (generando la secuencia agramatical *Muriéndose Juan tiene que haber estado...) y realizado también en posición de especificador de los auxiliares funcionales ser y haber (contra los datos de (21) y la observación de Torrego 1984 de que los auxiliares ser y haber no pueden preceder inmediatamente al sujeto en casos de inversión en interrogativas Qu-). Por otra parte, si el sujeto permanece en el dominio de SV / Sv en la anteposición inductora de verum focus como se ha sugerido que lo hace en los casos de anteposición de un operador (como el movimiento Qu-; ver nota 6), entonces no debería poder aparecer a la izquierda de un verbo léxico (si el verbo sube a $\mathrm{T} / \mathrm{Flex}$ ); en este caso la teoría resultaría inadecuadamente restrictiva ya que es posible tener ejemplos como (23):

(23) ¿Qué podría Juan haber estado haciendo?,

donde el sujeto aparece a la izquierda de dos auxiliares y del verbo léxico, pero a la derecha de un auxiliar léxico. Si, como sostienen Barbosa (2001), Uribe-Etxebarría (1992) o Zubizarreta (2001), entre otros, los sujetos en español no suben al especificador de T/Flex en las interrogativas, entonces no hay forma de generar la secuencia de (23) a menos que se multipliquen los movimientos a la derecha con escaso fundamento teórico o empírico.

Pero la anteposición de cada auxiliar como un núcleo no es la única opción posible, como vimos arriba. Si la cadena de auxiliares se antepone como un único objeto complejo, a su vez, tenemos dos opciones: (i) el primer auxiliar se lleva al sujeto a su izquierda, o (ii) el sujeto se queda en Espec-T y la cadena se mueve independientemente. Representemos ambas opciones (asumamos que $\mathrm{Y}^{0}$ es un núcleo funcional inductor de verum focus, posiblemente para cotejar un rasgo criterial en el sentido de Rizzi 2010; crucialmente, los problemas que señalamos surgen cualquiera sea la motivación que se asuma para la anteposición — cotejo de rasgos, satisfacción de requerimientos de estructura informativa, creación de relaciones Operador-Variable...-): 


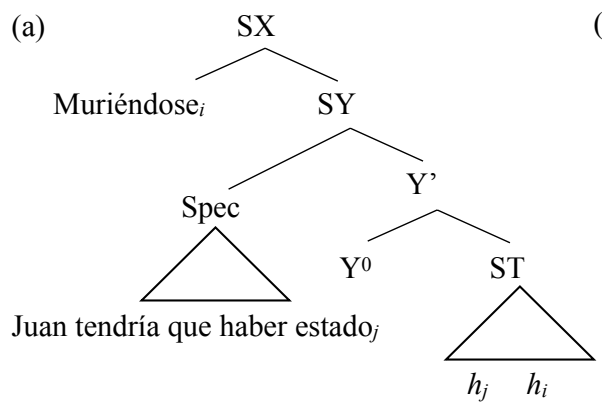

(b)

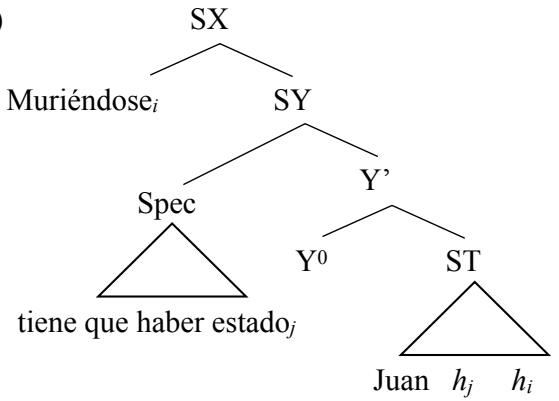

Evidentemente, (24a) debe ser excluida como derivación porque el orden que genera es agramatical, con el sujeto adyacente al gerundio. Por otro lado, (24b) genera un orden posible, aunque predice que la única posición en la que puede aparecer el sujeto es después del último auxiliar, lo cual resulta insuficiente ya que podemos tener sujetos internos a la cadena de auxiliares: en (24b) no hay sitio para que el sujeto aparezca a la derecha de tener, lo cual es no obstante perfectamente posible (cfr. Muriéndose tendría Juan que haber estado para no ir a esa fiesta). En resumen: es necesario que los auxiliares suban por encima del sujeto para evitar que el sujeto aparezca inmediatamente a la derecha del gerundio antepuesto (*Muriéndose Juan tendría que haber estado para no ir a esa fiesta), pero suponer que las cadenas de auxiliares son objetos internamente opacos (lo cual se seguiría de la idea de que es toda la cadena lo que auxilia al verbo léxico, que aparece en Gómez Torrego 1999: 3346, y Guéron \& Hoekstra 1988: 36-37, entre otros) resulta demasiado restrictivo. Igualmente, hay que evitar que la cadena de auxiliares se anteponga con un sujeto propio, como en (24a). De nuevo, en combinación con la idea de que la cadena ha de subir como objeto complejo, sostener que los sujetos aparecen en T/Flex o en Spec-V de manera uniforme resulta demasiado restrictivo: dejar al sujeto en SV permite generar únicamente el orden «Muriéndose tiene que haber estado Juan...», pero ninguno de los otros (en los que el sujeto aparece internamente a la cadena de auxiliares). Conviene recordar que, tanto por razones de orden de palabras como de alcance lógico, la cadena de auxiliares debe estar estructuralmente más alta que el SV).

Hemos visto que las dos opciones que se nos presentan en un modelo «tradicional» de estructura de frase (movimiento de núcleo a núcleo y movimiento a posición de especificador) resultan empíricamente insuficientes. Argumentamos aquí que la inadecuación de estos modelos se debe a que, cuando se asume que la computación sintáctica es uniforme (siempre binaria, basada en Ensamble, y creando únicamente 
unidades \{núcleo, Sintagma\}, como sostiene Chomsky 2009), la estructura interna de las cadenas de auxiliares (su organización alrededor de núcleos léxicos y dependientes funcionales y nominales) no puede ser adecuadamente representada.

Tenemos ya los elementos técnicos para terminar de exponer nuestra propuesta respecto de la posición de los sujetos: si las cadenas de auxiliares se estructuran en PPEE (que son, recordemos, unidades de predicación a nivel semántico y ciclos a nivel sintáctico; tienen de este modo entidad relativamente independiente en las «interfaces»), no es necesario «insertar» el sujeto después de la anteposición de cada $\mathrm{PE}$, porque siempre estuvo allí, dentro de la PE (y lo mismo sucede con los clíticos; ver en particular McCawley 1982, 1998; De Vries 2009; Citko 2014; Johnson 2016, entre otros). En este sentido, veamos cuál sería la estructura de cada PE para el ejemplo que venimos considerando, utilizando etiquetas familiares (y poniendo a los auxiliares en «SAux», por mera comodidad):

(25) (a)

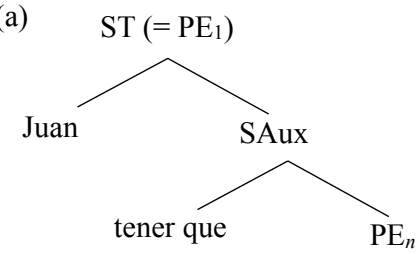

(b)

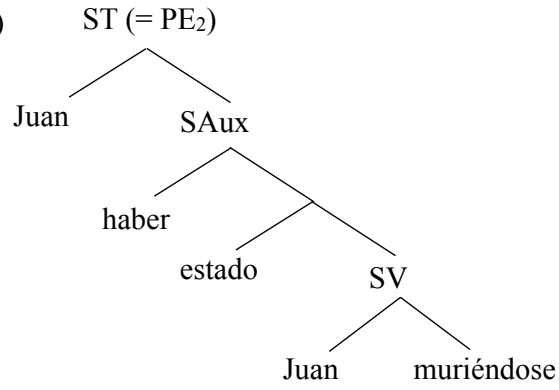

El complemento del auxiliar léxico tener que es una PE, que puede consistir simplemente de un verbo léxico con su sujeto (por ejemplo, en Juan tiene que trabajar); pero también puede contener auxiliares funcionales modificando al núcleo léxico de la PE, como es el caso de nuestra $\mathrm{PE}_{2}$. En la PE del verbo léxico, Juan aparece en dos posiciones: dentro del SV recibe rol temático y en ST recibe Caso. Hay que notar que cada PE contiene uno y solo un predicado léxico: en este sentido, cada PE es un «árbol elemental» en una gramática de adjunción (Tree Adjoining Grammar; Joshi 1985). Más específicamente, Frank $(1992,2002)$ propone una Condición de Minimidad de los Árboles Elementales que es formalmente equivalente a esta parte de nuestra propuesta, en tanto cada PE contiene solo un predicado léxico (un verbo pleno o un auxiliar léxico); el resto del material depende de este núcleo: «Cada árbol elemental consiste de la proyección extendida de un único predicado léxico» (Frank 1992: 53; traducción nuestra).

La representación de (25b) puede simplificarse eliminando ocurrencias si asumimos (como es usual en gramáticas de dependencias y en trabajos como McCawley 
1982; Levine 1985, y las referencias citadas arriba) que un elemento está dominado por todos los elementos que lo modifican (una asunción básica y fundamental en modelos como gramática de dependencias; ver Tesnière 1959; Osborne et alii 2011 demuestran que el poder generativo fuerte de las versiones «modernas» del Minimalismo se acerca más a una gramática de dependencias que a una gramática de estructura de frase): esto nos permite eliminar copias y huellas como primitivos en las derivaciones sintácticas ${ }^{9}$, al rechazar el axioma conocido como Condición de Madre Única (de acuerdo con el cual todo nodo puede tener como máximo un nodo madre, que lo domine; ver e.g. Sampson 1975). Estrictamente, entonces, (25b) debería reemplazarse por (25b'), ya que Juan es un elemento idéntico cuyas ocurrencias solo difieren en el contexto sintáctico en el que aparecen:

(25) b'

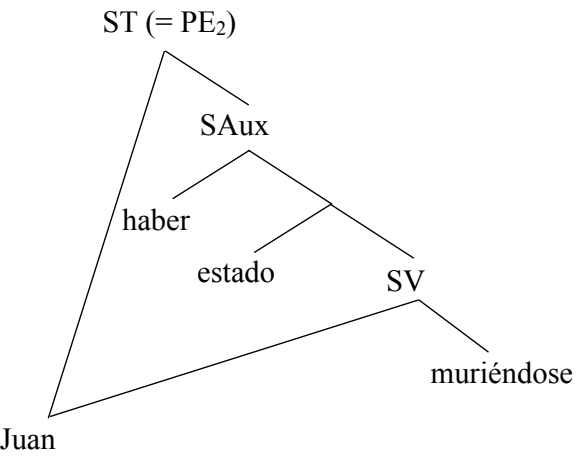

$9 \quad$ Las ventajas computacionales de las teorías de unificación, que tienen el mismo poder generativo fuerte que las de multidominancia, han sido estudiadas en detalle. Karttunnen y Kay (1985) demuestran que, en términos de complejidad, las teorías basadas en huellas o copias requieren un tiempo de análisis (parsing) super-polinómico (y ciertamente mayor que el tiempo de análisis requerido para identificar un mismo nodo en diferentes contextos, o, lo que es lo mismo, una misma matriz de rasgos en dos super-matrices unificadas, como en Shieber 1986). Además, resultan menos restrictivas en términos de las posibles dependencias que pueden establecerse: en principio, cualquier nodo (terminal o no) puede copiarse y moverse a cualquier otro nodo; las restricciones involucran supuestos adicionales (como la versión original de Preservación de Estructura en Emonds 1970; reinterpretada y debilitada en Chomsky 1986). A este respecto, De Vries (2009: 347-348) comenta - acertadamente en nuestra opinión - que «What should be clear is that the assumption of copies would require theoretical machinery in addition to the operation Merge per se» (recuérdese que Ensamble es una operación libre de contexto, mientras que la copia y re-Ensamble es al menos débilmente sensible al contexto). El desplazamiento como movimiento (copia y re-Ensamble) necesita de un procedimiento adicional de indexación (identificación de ocurrencias y repeticiones de un objeto sintáctico) y borrado de copias o huellas (por ejemplo, el propuesto en Lasnik \& Saito 1984 con el marcado $[\gamma]$ o «Affect- $\alpha$ », que incluye «delete anything anywhere — syntax and LF-》 (p. 258)) inicialmente irrestricto. Toda restricción resulta, por tanto, extrínseca a la operación tanto de creación de estructura — Ensamble — como de modificación de estructura — Movimiento—. 
Cuando las dos PPEE (que contienen un predicado léxico tener que y muriendo respectivamente) se relacionan mediante adjunción (en este caso, substitución), los elementos comunes a los árboles elementales (PPEE) se identifican: esta identificación es la que nos permite evitar la multiplicación de copias si aceptamos que un elemento puede estar dominado por más de un nodo. La estructura queda como en (26):

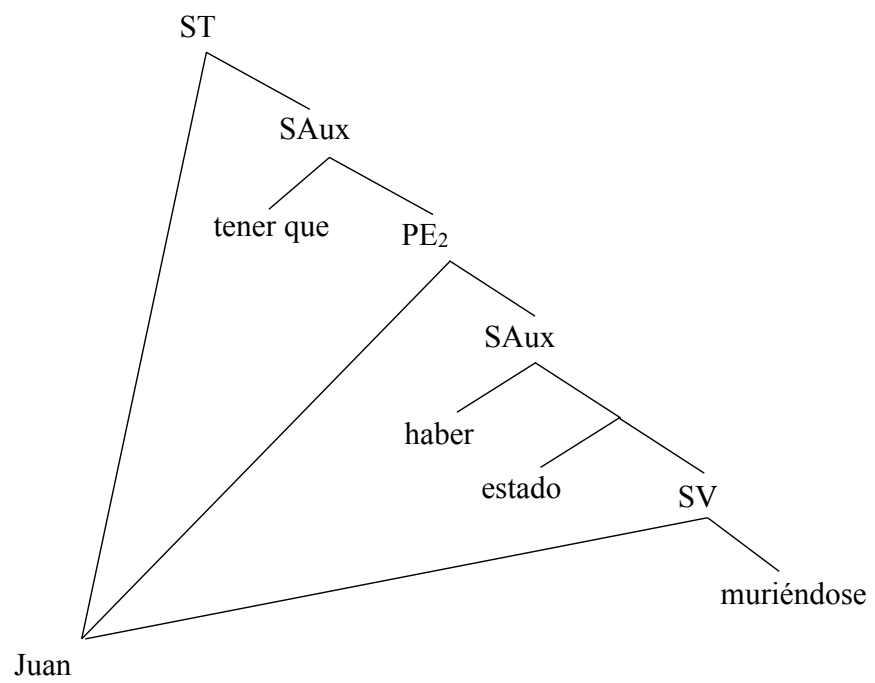

Vemos que Juan está dominado por la proyección de tener que y por la de muriéndose, pero no por haber ni por estado. La interpretación semántica y la materialización fonológica simplemente identifican instancias del mismo nodo en diferentes contextos estructurales.

La derivación de arriba ilustra cómo se genera la versión declarativa Juan tiene que haber estado muriéndose (omitiendo el adjunto a SV para simplificar el diagrama y porque su omisión es inconsecuente). A continuación, trataremos la versión con anteposición inductora de verum focus. Primeramente, el gerundio se antepone a una posición que simplemente llamaremos $\mathrm{C}$ y esta focalización (opcional) tiene efectos análogos a la interrogación: motiva la subida (obligatoria, como vimos en (5) y (18)) de la cadena de verbos auxiliares que modifica a este gerundio, subida que se da por PPEE: hay que tener en cuenta, como hemos ya señalado, que las PPEE se adjuntan por debajo de la raíz. La raíz domina inmediatamente al gerundio y la cadena de auxiliares se adjunta por PPEE entre el gerundio y SFlex/ST. Vamos a ilustrar la derivación pertinente. 
El primer paso es la anteposición del gerundio, que podemos simplemente expresar como $\mathrm{C}$ dominando al gerundio (en lugar de mover el gerundio a $\mathrm{C}$ y dejar una huella en la posición de núcleo de SV; esta operación requiere, además, de un algoritmo de indexación e identificación independiente de Ensamble y Movimiento a los efectos de construir una representación adecuada en Forma Lógica):

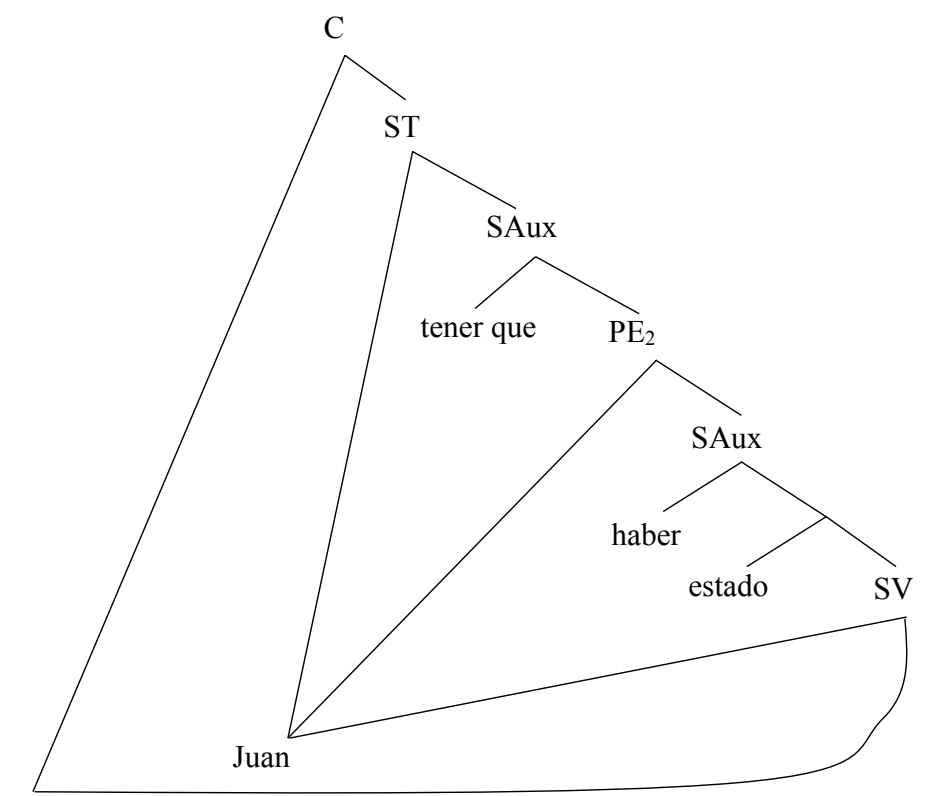

muriéndose

Esto es importante, porque, como vimos en la cita de Frank (1992), en una gramática de adjunción lexicalizada cada árbol elemental está estructurado alrededor de un único núcleo léxico: el árbol elemental de C, por lo tanto, debe ser como en (28), con el gerundio actuando como núcleo léxico del sub-árbol:

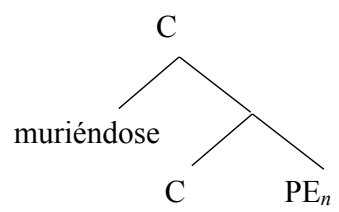


Como muriéndose está dominado en un árbol inicial por $\mathrm{C}$ y en otro por $\mathrm{SV}$, cuando substituimos PE en el árbol de (28) por el árbol completo de (26), simplemente identificamos las dos instancias del gerundio y lo convertimos en un nodo multidominado (o, lo que es equivalente, unificamos ${ }^{10}$ en el sentido de Shieber 1986): así llegamos a (27). A continuación, tenemos que considerar la adjunción de PPEE por debajo de $C$ pero por encima de $T$ (para evitar la generación del orden agramatical *Muriéndose Juan tiene que...). Aquí es donde la propuesta de Joshi (1985) de adjunción de árboles resulta particularmente útil. Joshi define una operación de adjunción que se aplica a sub-árboles de la siguiente manera:

(...) compone un árbol auxiliar $\beta$ con un árbol $\gamma$. Sea $\gamma$ un árbol con un nodo etiquetado como $\mathrm{X}$ y sea $\beta$ un árbol auxiliar cuya raíz también está etiquetada como $\mathrm{X}$ (nótese que $\gamma$ debe tener, por definición, uno y solo un nodo etiquetado como $\mathrm{X}$ en su frontera) (Joshi 1985: 209; traducción nuestra).

Por ejemplo, veamos el resultado de adjuntar un árbol auxiliar en un árbol inicial, donde $\mathrm{X}=$ Aux y manteniendo las letras griegas usadas por Joshi para identificar los sub-árboles involucrados en la operación:
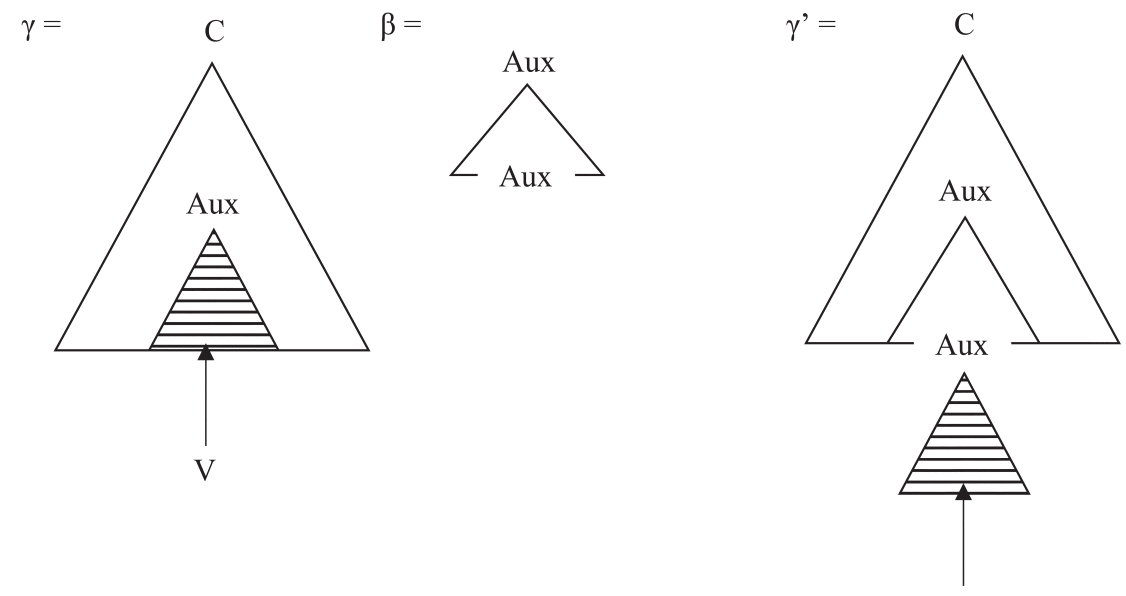

V

10 «In formal terms, we define the unification of two feature structures D and D' as the most general feature structure D, such that D' $\subseteq$ D and D' $\subseteq$ D. We notate this D = D' $\cup$ D'» (Shieber 1986: 14). De este modo, el algoritmo se aplica como sigue:

i) Unificar $(<\mathrm{C}$, muriéndose $>,<\mathrm{V}$, muriéndose $>)=(<\mathrm{C}, \mathrm{V}$, muriéndose $>)$

(por el axioma de par de teoría de conjuntos, $<\mathrm{C}$, V, muriéndose $>=<\mathrm{C},<\mathrm{V}$, muriéndose $>>$, que es un conjunto ordenado. La relación de orden, en el caso que nos ocupa, es dominancia; ver McCawley 1968; Zwicky \& Isard 1963; Krivochen 2018, para detalles formales). 
Esto evita tener que proponer una serie indeterminada y arbitraria de posiciones entre $\mathrm{T}$ y $\mathrm{C}$ donde puedan aterrizar los constituyentes para los cuales se vaya a proponer un desplazamiento. Cuando se antepone una cadena de auxiliares, cada auxiliar léxico con todos sus dependientes (es decir, cada PE) corresponde a un sub-árbol, que en (25) sería el árbol inicial $\beta$. Estos sub-árboles se adjuntan por encima de SFlex/ST, pero por debajo del nodo raíz (es decir, el nodo más alto, C en (29)) y, en este proceso, SFlex/ST se «desplaza» hacia abajo, como ocurre en (25) con el sub-árbol sombreado. Esto es posible porque el árbol adjunto contiene un nodo idéntico a la raíz del árbol desplazado (en (25), la raíz del árbol sombreado es Aux y el árbol inicial $\beta$ contiene un nodo Aux en su «frontera» inferior; esta identidad permite la adjunción no-cíclica). Como indicamos arriba, nuestros sub-árboles son las PPEE de los núcleos léxicos (ver también Frank 1992, 2002). Concretamente, lo que tenemos es:

(30) Arboles elementales:

(a)

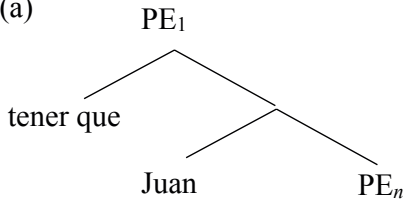

(b)

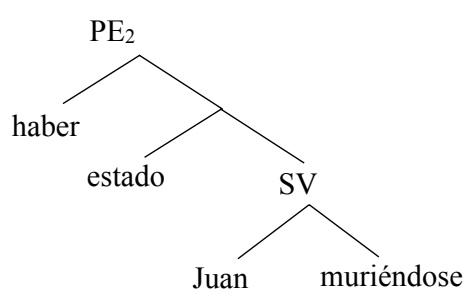

Como vimos en (27), el complemento de $\mathrm{C}$ es siempre una PE: incluso en términos generativos tradicionales, SC toma como complemento a ST (o SFlex), que es la PE del SV (Grimshaw 2005; Abney 1987). La situación, entonces, es la de (30), en la que la adjunción de la cadena de auxiliares por encima de T/Flex (lo cual está legitimado por el hecho de que el complemento del árbol inicial $\mathrm{C}$ selecciona un complemento T/Aux), «desplaza» las PPEE 1 y 2 hacia abajo (en lo que sigue, el lado en el que aparecen los elementos en el árbol no es formalmente significativo, siendo que los árboles son grafos en el sentido técnico; Zwicky \& Isard 1963; Krivochen 2018): 
(31)

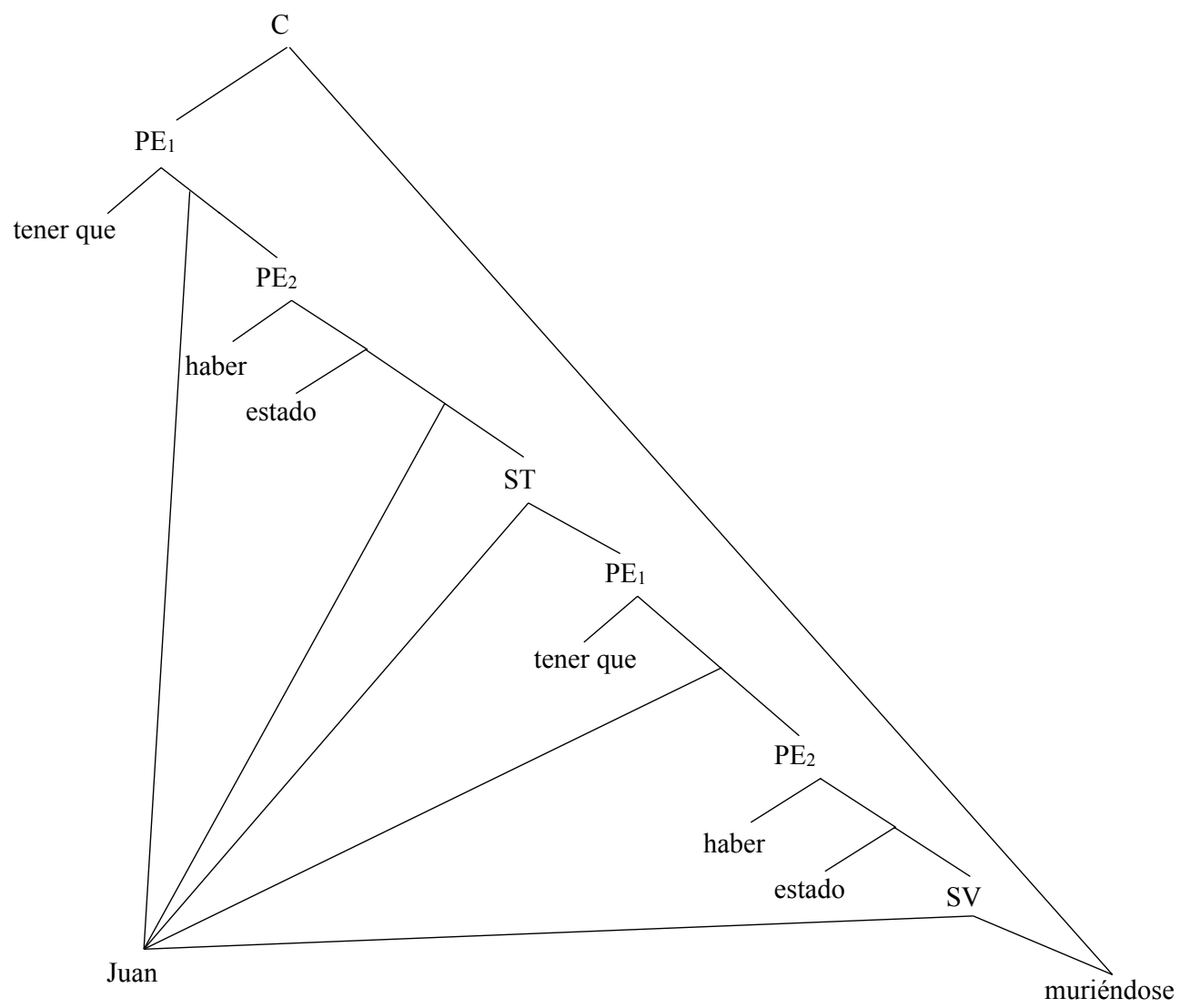

Vayamos por partes: tenemos un nodo Juan, que está dominado por varios otros nodos, y cada instancia debe estar justificada. Juan dominado por SV recibe rol temático. Juan dominado por T recibe Caso Nominativo. Tener que es un auxiliar léxico, que toma a Juan como uno de sus argumentos en virtud de heredar la estructura-A del verbo léxico pleno morirse: recordemos que las PPEE que modifican al verbo léxico pleno heredan su estructura-A porque forman parte de la proyección-s de éste (Abney 1987). Las ocurrencias por encima de T están licenciadas por el hecho de que simplemente estamos re-adjuntando estructura que ya está presente en la derivación (y, estrictamente hablando, deberíamos tener más nodos multidominados en lugar de repetir auxiliares, pero lo hemos hecho estrictamente para evitar que el diagrama resulte más confuso). Crucialmente, el diagrama (31) captura las dependencias sintácticas 
y semánticas que tenemos en el ejemplo, sin asignar más estructura de la requerida (es decir, sin recurrir a nodos terminales en la forma de huellas, o no terminales en la forma de proyecciones funcionales ad hoc ajenas tanto al input como al output de las operaciones pertinentes) y restringiendo adecuadamente las posibilidades de realización de los sujetos internos a la cadena de auxiliares, que, recordemos, no pueden aparecer a la derecha del gerundio pero sí en posiciones entre los auxiliares, incluyendo a la derecha del último.

Empíricamente, la derivación no difiere en esencia respecto de lo que sucede en el movimiento Qu-. Consideremos (32):

(32) a. Juan podría haber tenido que leer ese libro.

b. ¿Qué libro (*Juan) podría (Juan) haber (*Juan) tenido (Juan) que leer?

En este caso, vemos que hay movimiento del SQu- qué libro a Spec-C (que aquí representamos de manera equivalente, mediante un nodo qué libro multidominado por $\mathrm{V}$ y por C) y la cadena de auxiliares debe anteponerse, pero no es posible tener el SQuadyacente al sujeto Juan (como vemos en (32b)). El movimiento de un elemento a una posición en la periferia de $\mathrm{C}$ motiva la anteposición no del auxiliar estructuralmente más alto, sino de toda la cadena, presentando el mismo patrón empírico que los casos de verum focus que hemos analizado aquí: es posible tener el sujeto materializado internamente a la cadena, aunque no de manera completamente libre.

Para (32), los árboles iniciales y la representación final son los siguientes:
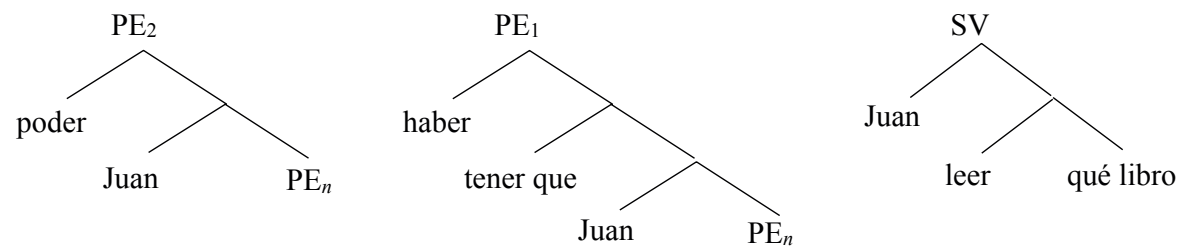


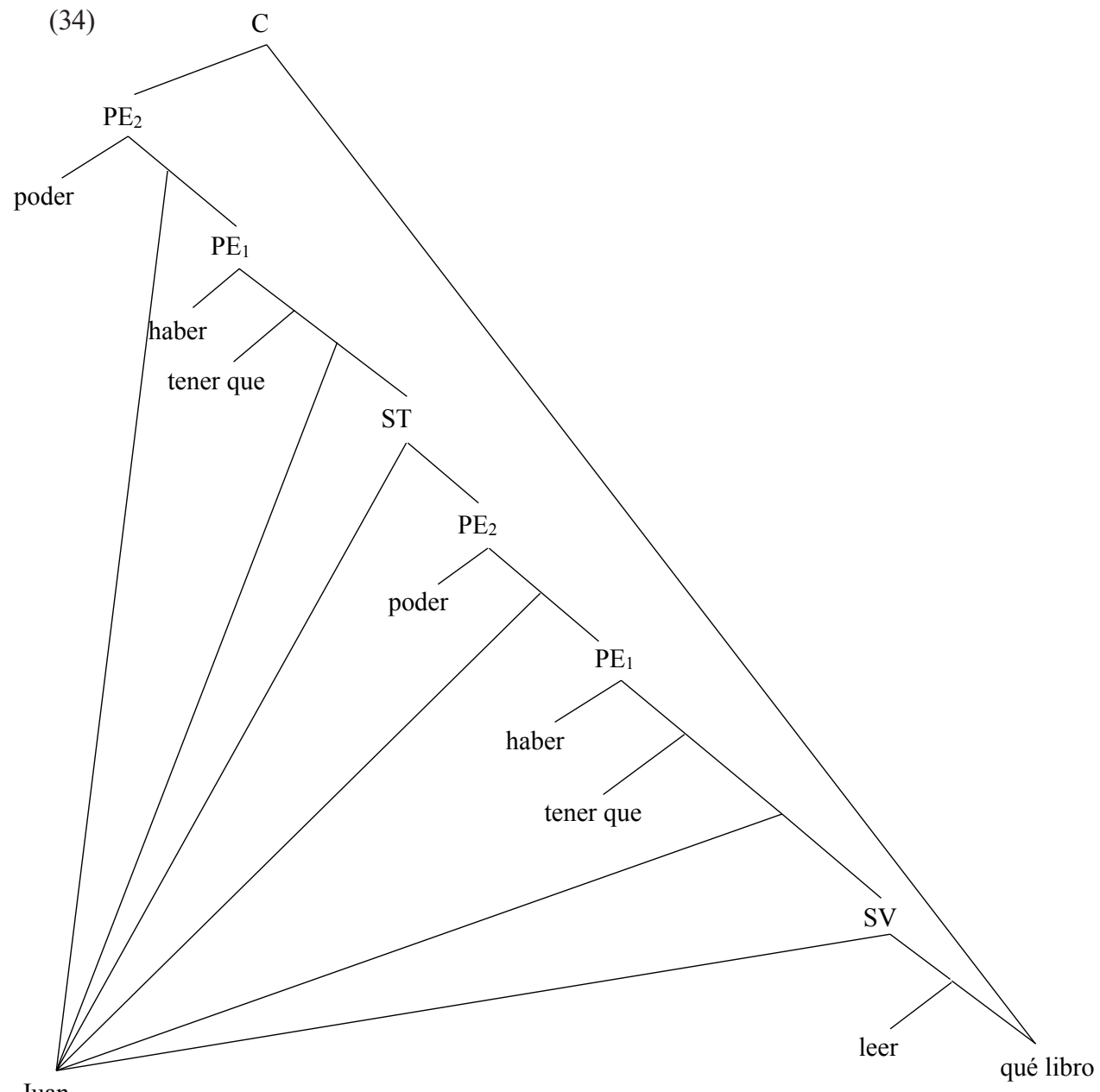

Juan

Las restricciones respecto de la posición de los sujetos también acercan el verum focus y el movimiento Qu-. La anteposición de cadenas de auxiliares por PPEE predice que la aparición de sujetos en posiciones adyacentes a auxiliares funcionales resultará menos aceptable (dependiendo del hablante, muy marcada, marginal, o directamente agramatical), ya que los sujetos son dependientes del núcleo léxico de la PE. De este modo, (18) — repetido aquí- resulta paralelo a (35): 
(18) Muriéndote (*tú) debes (tú) haber (\%tú) tenido (tú) que estar para no venir al examen.

(35) ¿A quién (*Juan) tiene (Juan) que haber (*Juan) estado (Juan) pidiéndole perdón?

La estructuración de las cadenas de verbos auxiliares en torno a núcleos léxicos y la distinción entre auxiliares léxicos y funcionales en el sentido de Bravo et alii (2015) y García Fernández et alii (2017) ofrece ventajas teóricas y empíricas, entre las cuales se encuentra la potencial unificación de algunos mecanismos sintácticos para la derivación de verum focus y movimiento-Qu.

\section{VERUM FOCUS Y SUBIDA DE CLÍTICOS}

Pasemos ahora a ocuparnos de la segunda cuestión que hemos anunciado: la interacción entre la anteposición cuyos mecanismos hemos descrito en la sección anterior y la subida de clíticos.

Retomemos el ejemplo (8a), repetido aquí:

(36) Escuchándote estoy.

Ya hemos visto que esta secuencia puede obedecer lógicamente a dos segmentaciones, que repetimos:

(37) a. [Forma no finita + clítico] + auxiliar

b. [Escuchándote] estoy.

(38) a. Forma no finita $+[$ clítico + auxiliar $]$

b. Escuchando [te estoy].

Como hemos anunciado, la segmentación de (38) no es posible. Empíricamente se puede demostrar que es así con la inserción de la negación ${ }^{11}$.

(39) a. Escuchándote no estoy.

b. *Escuchando no te estoy.

$11 \quad$ Leonetti y Escandell Vidal (2009: 193) sostienen que las construcciones con anteposición inductora de verum focus son incompatibles con la negación, lo cual prima facie podría invalidar el razonamiento basado en (39) y (40) (como nos ha sugerido un revisor anónimo). No obstante, Leonetti y Escandell Vidal (2009) consideran únicamente anteposición de SN; de este modo, la incompatibilidad entre la negación y la anteposición inductora de verum focus se explica diciendo que «in negative sentences definites tend to be interpreted as referential expressions, falling outside of the scope of negation» (2009: 193). Pero este no es el caso cuando lo antepuesto es el núcleo de SV. 
(40) a. Hacerlo no podría.

b. *Hacer no lo podría.

En los ejemplos de (39a) y de (40a) tenemos un verbo léxico auxiliado que se ha antepuesto. En ambos casos tiene un pronombre enclítico y le sigue la negación; en ambos casos el resultado es gramatical. Por el contrario, en (39b) y en (40b), la negación está delante del clítico y este está proclítico al verbo auxiliar en forma finita; el resultado es agramatical.

Lo mismo sucede con la inserción del adverbio ya:

(41) a. Escuchándote ya estoy.

b. *Escuchando ya te estoy.

(42) a. Hacerlo ya podría.

b. *Hacer ya lo podría.

Y, evidentemente, el contraste se repite de nuevo si insertamos la negación y ya:

(43) a. Escuchándote ya no estoy.

b. *Escuchando ya no te estoy.

(44) a. Hacerlo ya no podría.

b. *Hacer ya no lo podría.

Estos contrastes muestran que, en la secuencia $<$ forma no finita + clítico + auxiliar>, el clítico es siempre enclítico a la forma no finita. La cuestión que debemos explicar es por qué.

A efectos descriptivos, podemos identificar dos reglas involucradas en la derivación de ejemplos como (45):

(45) Escuchándote estoy.

I. Una regla que forma una terminal léxica ${ }^{12}$ a partir de una configuración $\mathrm{V}+$ Clítico, que llamaremos apoyo de clítico.

II. Una regla radical de anteposición (en el sentido de Emonds 1970: 10).

12 En el análisis de lenguajes formales se distingue entre elementos terminales y no terminales (e.g., Hopcroft \& Ullman 1969: 10). Solo los no terminales aparecen a la izquierda de las reglas de reescritura, es decir, son nodos que ramifican (por ejemplo, «SV», « $\mathrm{SN} »)$. Los nodos terminales no dominan a ningún otro nodo (por ejemplo, $\mathrm{N}, \mathrm{V}$ ). 
Nos hemos ocupado de las propiedades estructurales de (II) en la sección anterior, aquí nos ocuparemos de especificar las condiciones bajo las cuales se puede aplicar (I). Es interesante observar cómo interactúan estas reglas con la de subida de clíticos $^{13}$.

La formulación de subida de clíticos asume necesariamente que el clítico es visible para la sintaxis: esto significa que la descripción estructural que debe satisfacerse es (46a) y no (46b), abajo. En (46a) el clítico no forma una terminal léxica con el $\mathrm{V}$ y por lo tanto es visible para la sintaxis; en (46b) el clítico se ha incorporado al $\mathrm{V}$ formando una terminal léxica, la sintaxis ya no puede por tanto manipular el clítico como una unidad independiente (ver Bleam 2000: 194 para una condición similar: para ser manipulados por una regla sintáctica, los clíticos deben ser unidades independientes al momento de aplicar dicha regla):

(46) a. $\mathrm{X}+\mathrm{V}_{\mathrm{Ger}}+\mathrm{Cl}$ (e.g., escuchando te) $\rightarrow$ Antes de «apoyo de clítico» b. $\mathrm{X}+\# \mathrm{~V}_{\mathrm{Ger}}+\mathrm{Cl} \#$ (e.g., escuchándote) $\rightarrow$ Via «apoyo de clítico»

Donde $\mathrm{X}$ es una variable sobre secuencias de auxiliares (por ejemplo, puede adoptar el valor «Puedo haber tenido que estar»), + es concatenación lineal y \# establece límites entre terminales a los efectos de la computación sintáctica (Chomsky 1957: 39, nota 5). Si (46b) está derivacionalmente relacionada con (46a), llamaremos al mapeo desde (46a) a (46b) apoyo de clítico, y descriptivamente lo formulamos como sigue:

(47) Apoyo de clítico: \#V\#+Cl $\rightarrow\left\{\begin{array}{l}a . \# V_{[-F i n]}+C l \# \\ b . \# C l+V_{[+F i n]} \#\end{array}\right\}$

Esto simplemente quiere decir que, en una secuencia de verbo más clítico, el clítico aparece como proclítico si el verbo es finito (condición contextual (b): \#hago\# + lo = \#lo hago\#) y como enclítico si el verbo no es finito (infinitivos y gerundios, condición (a): \#hacer\# + lo = \#hacerlo\#), en los límites de una terminal sintáctica demarcada con \#. Cabe destacar que (47) no es una regla de movimiento, sino de formación de una terminal léxica (una regla morfo-fonológica) ${ }^{14}$.

13 Conviene recordar que en una gramática máximamente explícita (uno de los sentidos en los que se entiende generativa) todas las operaciones deben especificar tanto su input como su output: la caracterización de una regla sintáctica debe incluir la clase de estructuras a las que se aplica (su input, lo que se conoce como descripción estructural) y la clase de estructuras que genera (su output, lo que se conoce como cambio estructural). Esta especificación es fundamental en el desarrollo de una gramática formal empíricamente adecuada; las reglas abstractas del tipo Muévase- $\alpha$ no escapan a este requerimiento de explicitud (como se puede ver en el título de Lasnik \& Saito 1992). Desde los '70 hasta el día de hoy ha cambiado la forma de especificar el input y el output de las reglas, pero el requerimiento de completa explicitud permanece intacto.

14 Es necesario notar que la regla que hemos formulado descriptivamente considera verbos conjugados, infinitivos y gerundios. A los efectos de (47) no hemos considerado los imperativos. 
En este punto, debemos tener en cuenta tres reglas: apoyo de clítico, subida de clítico y anteposición. Analicemos ahora cómo interactúan. Obviamente, la aplicación de apoyo de clítico impide la de subida de clíticos, ya que subida de clítico requiere, como acabamos de señalar, que el clítico sea visible para la sintaxis; es decir, que sea un nodo terminal independiente. No obstante, la regla de anteposición que coloca un elemento en la periferia izquierda (y que ha sido el objeto de análisis en la sección anterior) debe aplicarse luego de apoyo de clítico cuando el clítico se aloja en el gerundio (es decir, una vez que tenemos una configuración como la de (46b)). El concepto de orden entre reglas depende crucialmente de que las reglas que han de ser ordenadas puedan aplicarse en la misma derivación (Kiparsky 1968; Ringen 1974) ${ }^{15}$. Si un clítico forma una palabra fonológica con otro nodo mediante (47), entonces no puede ya subir porque la regla de subida no tiene acceso a ese clítico; esto implica que apoyo de clítico, de aplicarse en una derivación en la que se ha aplicado subida de clíticos, debe hacerlo post-cíclicamente. Es decir: primero el clítico sube hasta donde tenga que subir y luego se aloja en un huésped apropiado mediante (47).

Nuestra propuesta respecto de la interacción entre verum focus y subida de clíticos tiene dos partes.

Por un lado, proponemos que hay un ordenamiento intrínseco (Koutsoudas \& Sanders 1979; Ringen 1974) entre las reglas de apoyo de clítico y anteposición, de modo tal que:

(48) Apoyo de Clítico > Anteposición (léase: 'Apoyo de Clítico se aplica antes que Anteposición')

$\mathrm{Y}$, en este ordenamiento, anteposición «contra-sangra» ${ }^{16}$ apoyo de clítico, en el siguiente sentido: la aplicación de anteposición restringe la posibilidad de aplicar

15 El concepto de orden es fundamental en la construcción de una gramática explícita, tanto en modelos basados en teoría de la demostración (proof theory) como en teoría de modelos (model theory) (ver Pullum \& Scholz 2001 para una comparación entre estos dos enfoques). En un marco minimalista ortodoxo, Epstein \& Seely (2006: 70, ss.) y Chomsky (2001: 5) discuten los problemas inherentes al ordenamiento de las operaciones de Concordancia y Materialización (aunque sin la sistematicidad que encontramos en Ringen 1974 o Koutsoudas \& Sanders 1979).

16 La noción de «contra-sangrado» en el ordenamiento entre reglas sintácticas es expuesta en Koutsoudas et alii (1974: 2) como sigue:

si A se aplica antes que B, B entrará en una de las siguientes relaciones con A:

(a) B CONTRA-ALIMENTA a A si y solo si la aplicación de B AUMENTARÍA el número de formas a las que A podría aplicarse SI B fuera a aplicarse antes que A.

(b) B CONTRA-SANGRA a A si y solo si la aplicación de B DISMINUIRÍA el número de formas a las que podría aplicarse A SI B se fuera a aplicarse antes que A.

(c) B NO AFECTA a A si y solo si B ni contra-alimenta ni contra-sangra a A. (Koutsoudas et alii 1974: 2; traducción nuestra). 
apoyo de clítico, porque si anteponemos el gerundio se pierde la adyacencia estructural necesaria para que se satisfaga la descripción estructural de apoyo de clítico en (47). Esto implica que en un caso como:

(49) Escuchándote estoy,

la derivación correspondiente es (50), enriquecida con huellas e índices a efectos puramente ilustrativos:

(50) A. $\left[_{\mathrm{ST}}\right.$ pro Estoy $\left[{ }_{\mathrm{SV}}\right.$ escuchando te $\left.]\right]$

B. $\left[_{\mathrm{ST}}\right.$ pro Estoy $\left[_{\mathrm{SV}}\right.$ escuchándote $\left.]\right] \rightarrow$ via apoyo de clítico

C. $\left[{ }_{\mathrm{SX}}\right.$ escuchándote $_{i}\left[{ }_{\mathrm{X}}\right.$ estoy ${ }_{j}\left[_{\mathrm{ST}}\right.$ pro $\left.\left.h_{j}\left[{ }_{\mathrm{SV}} h_{i}\right]\right]\right] \rightarrow$ via anteposición

En relación al paso (50c), y como segundo aspecto de nuestra hipótesis, proponemos analizar la anteposición del gerundio como un caso de verum focus. Consideremos, por ejemplo, (51):

(51) *Engañando lo puede estar toda la vida.

El caso (51) queda adecuadamente excluido a partir del ordenamiento de reglas que hemos propuesto en (48) y su interacción con la regla de subida de clíticos. Obsérvese que la derivación de (51), en (52) abajo, aplica el orden inverso al de (48), resultando en agramaticalidad:

(52) A. $\left[_{\mathrm{ST}}\right.$ pro puede estar [ ${ }_{\mathrm{SV}}[\mathrm{SV}$ engañando lo] toda la vida] $]$

B. $\left[_{\mathrm{SX}}\right.$ Engañando ${ }_{i}\left[_{\mathrm{ST}}\right.$ pro puede estar $\left[{ }_{\mathrm{SV}}\left[{ }_{\mathrm{SV}} h_{i} l \mathrm{l}\right]\right.$ toda la vida $\left.]\right] \rightarrow$ via anteposición

C. $\left[{ }_{\mathrm{SX}}\right.$ Engañando ${ }_{i}\left[_{\mathrm{ST}}\right.$ pro $l_{j}$ puede estar $\left[{ }_{\mathrm{Sv}}\left[{ }_{\mathrm{SV}} h_{i} h_{j}\right]\right.$ toda la vida $\left.]\right] \rightarrow$ via subida de clítico

La condición abstracta relevante es, a nuestro juicio, que el elemento anteponiendo no puede formar parte de una cadena $C=\left(X_{i}, h_{i}\right)$, donde X es el elemento antepuesto, si hay un clítico (o una cadena que incluya un clítico) estructuralmente entre $X_{i} y$ su huella $h_{i}$. Esta condición respecto de la integridad de las cadenas retoma aspectos de las condiciones en Forma Lógica propuestas en May (1985) y Sloan (1991), y en general desfavorece las dependencias cruzadas en la formación de cadenas mediante movimiento (pero no necesariamente mediante reglas de estructura de frase o transformaciones generalizadas como sustitución o adjunción; ver Joshi 1985). Este es un punto importante, porque une el fenómeno de verum focus como lo presentan Leonetti y Escandell Vidal (2009) con la anteposición de gerundio que analizamos aquí y nos 
provee de un argumento más para decir que, efectivamente, el proceso de anteposición de gerundio es una instancia de anteposición inductora de verum focus. Veamos a este respecto las estructuras abstractas de (4) («* poco te lo puedo decir») y (51):

(4') $\mathrm{X}_{i} \ldots \mathrm{Cl}_{i} \ldots h_{i}$ (e.g., ${ }^{*} \mathrm{Poco}_{i}$ te $l o_{i}$ puedo decir $h_{i}$ )

(51') $\mathrm{X}_{i} \ldots \mathrm{Cl}_{j} \ldots h_{i} h_{j}$ (e.g., *Engañando $\operatorname{lo}_{j}$ puede estar $h_{i} h_{j}$ toda la vida)

La misma condición abstracta que excluye los casos de anteposición de gerundio sin que primero se haya aplicado apoyo de clítico excluye también (como es deseable) los casos de anteposición inductora de verum focus con pronombre reasuntivo: en ambos casos, el elemento antepuesto forma parte de una cadena que se ve interrumpida por potenciales antecedentes para la huella en posiciones no incrustadas (que corresponden a la estructura abstracta ...i...j...j...i...), sino cruzadas (el patrón ...i...j...i...j que vemos en $\left.\left(45^{\prime}\right)\right)$. Las condiciones comunes en el ordenamiento de las reglas y las configuraciones que pueden resultar, que han sido el foco de esta sección, proporcionan argumentos para la identificación de la anteposición de gerundio en las estructuras que nos interesan como una instancia de verum focus.

El ordenamiento de reglas en (48) también nos permite dar cuenta de paradigmas como el de (53):

(53) a. Engañándole puede llevar toda la vida.

b. *Engañando puede llevarle toda la vida.

c. Diciéndolas puede estar toda la tarde.

d. *Diciendo puede estarlas toda la tarde.

Los casos (53a) y (53c) se derivan mediante clitización al gerundio en un punto muy temprano de la derivación (tan pronto como la descripción estructural requerida por la regla de apoyo de clítico sea satisfecha) y la posterior anteposición de la terminal \#Gerundio+Clítico\# («engañándole», «diciéndolas»). El ordenamiento de (48) no permite que la sintaxis manipule el clítico independientemente una vez que ha formado una terminal léxica con el gerundio; de esta manera, subida de clíticos queda bloqueada en una derivación en la que se ha aplicado clitización. La derivación de (53b) y (53d) requiere que la sintaxis tenga acceso al clítico para «subirlo» hasta un auxiliar por encima de SV; esto implica que clitización no puede haberse aplicado, ya que el gerundio se ha antepuesto pero el clítico queda in situ (lo cual es imposible si ambos forman una terminal léxica).

Hemos argumentado que lo que tenemos, entonces, es la anteposición de una unidad léxica \#Gerundio + Clítico\#, que se mueve a la periferia izquierda e induce un 
verum focus. La aplicación de esta operación de anteposición incluye la anteposición del verbo léxico y sus auxiliares si los hubiere. No obstante, hemos de notar que en el caso en el que tenemos una cadena de auxiliares $\left[\operatorname{Aux}_{1} \ldots \operatorname{Aux}_{2} \ldots \mathrm{Aux}_{n} \ldots \mathrm{V}\right]$ la cadena no se mueve ni núcleo por núcleo (como en (22)) ni como una entidad única (como en (24)): lo que proponemos es que se antepone por PPEE.

En el caso que nos interesa, tenemos que explicar cómo puede crearse un lugar estructural para la cadena de auxiliares por encima de ST: esto no resulta problemático en una gramática de adjunción de árboles, ya que podemos tomar el sub-árbol que tiene a T como raíz y «correrlo» hacia abajo (como le ocurre al sub-árbol marcado con líneas horizontales en (29), arriba), adjuntando la cadena por encima. Las condiciones relevantes son:

I. Que el sitio de adjunción contenga una especificación que coincida con la raíz del sub-árbol que se vaya a adjuntar: en este caso, lo que puede subir a «C» en español es «Aux»/ «V».

II. Que la frontera del elemento adjunto contenga un elemento idéntico a la raíz del sub-árbol «corrido»: en este caso, lo que importa es que ST/SFlex es un dominio clausal y los auxiliares pueden tomar como complemento un dominio con estas características (que incluya predicados y argumentos, de la misma manera que un auxiliar toma como complemento al SV, que incluye en estadios tempranos de la derivación tanto al verbo léxico como a sus argumentos internos y externo).

Creemos que merece la pena aportar algunos ejemplos de los que habitualmente no se habla en la bibliografía; se trata de casos no demasiado comunes en los que tenemos repetición del clítico, en algunos casos en coocurrencia con doblado. Lo que nos permite el aparato teórico que hemos desarrollado aquí, que combina un sistema con multidominancia con la adjunción cíclica de PPEE, es dar cuenta de casos en los que el clítico puede aparecer en más de una $P E$ sin multiplicar copias:

(54) yo te iba a preguntarte... (ejemplo escuchado en la radio en España. Antonio Fábregas, c.p.)

Hemos dicho más arriba que la estructura-A del verbo léxico se legitima en cada PE que lo modifica debido a la estructura de los sub-árboles en una gramática de adjunción lexicalizada; las PPEE se estructuran alrededor de núcleos léxicos que son modificados por sus dependientes nominales y auxiliares funcionales. Los dependientes nominales 
tienen un nodo madre en cada PE en la que son seleccionados por un predicado léxico: son, hemos visto, nodos multidominados. La motivación básica, recordemos, es común en las gramáticas de dependencias: la «dependencia» es esencialmente una relación nodo madre-nodo hija (donde un nodo es la madre de otro si y solo si lo domina; ver e.g., Osborne 2008: 1122). De este modo, propusimos que, si un elemento establece una dependencia con más de un predicado, este elemento está dominado por todos los predicados pertinentes; el resultado es un modelo restrictivo que no multiplica las entidades sintácticas, sino las conexiones (un modelo para algunos de los fenómenos sintácticos principales en inglés en estos términos se presenta en detalle en Krivochen 2018). La adopción de este sistema predice que, en los casos en los que aparece el clítico repetido, como (54), los clíticos necesariamente comparten índice (lo cual, en una teoría con movimiento y huellas/copias, requeriría un mecanismo extra de co-indización, como el propuesto en Lasnik y Saito 1992 de co-indización libre en Forma Lógica): la PE de la que dependen es la PE del verbo léxico preguntar, ya que ir a es un auxiliar funcional y por lo tanto predecimos que dependa de la PE de preguntar. Ejemplos con clíticos repetidos son frecuentes en el español de Centroamérica, aunque no sean completamente ajenos al español de España:

(55) a. Aunque mi agenda me dice que hoy te tengo que decirte lo mucho que TE QUIERO (http://www.minglano.es/frases-para-felicitar-a-mama.htm - web radicada en España)

b. Te voy a quitar esas ganas, de estar conmigo / te voy a decirte también... (Letra de Somos los dos, de Fabián Corrales - Colombia)

c. Óyeme!... Te enteraste? Te voy a decirte a ti... (Letra de Sabor, Swing, y Sahoco, de V. M. Mairena - Costa Rica)

d. ...te voy a tener que decirte como el josep lluis a la señora que se empeñaba en llamarle jose luis (http://allegramag.info/recursos-sanitarios/ - web radicada en España, variedad de Cataluña)

Un análisis detallado de estas estructuras queda fuera del alcance del presente trabajo, aunque creemos que el sistema propuesto aquí resulta prometedor en la descripción de estos fenómenos. Lo que tenemos en estos casos es la realización morfo-fonológica del dependiente clítico del verbo léxico dentro del SV y en un auxiliar que modifica a este verbo léxico $(\mathrm{a}-\mathrm{b}, \mathrm{d})$ y un caso de doblado de clítico + repetición de clítico (c). 


\section{CONCLUSIONES}

En este trabajo hemos identificado una instancia de anteposición en español que reúne las propiedades para ser considerado un tipo de verum focus en el sentido de Leonetti y Escandell Vidal (2009). Esta anteposición afecta a gerundios que pueden o no tener un dependiente clítico y motiva la inversión de los verbos auxiliares que modifican al gerundio y el sujeto de la cláusula. Nuestra argumentación se ha focalizado en dos puntos:

a) Derivacionalmente, la operación que desplaza al gerundio a una posición por encima de Flex en la periferia izquierda es posterior a la clitización de un pronombre al gerundio; además, el movimiento se ve bloqueado si antes se ha aplicado una regla de subida de clítico. En este sentido, hemos reincorporado nociones conocidas respecto de las propiedades de orden entre operaciones en modelos derivacionales.

b) Aspectos del posicionamiento de los sujetos en las estructuras con anteposición de gerundio nos han ayudado a entender el tipo de operación que deriva el movimiento de auxiliares por debajo del gerundio y por encima de Flex. En primer lugar, siguiendo el modelo propuesto en Bravo et alii (2015), hemos argumentado que los auxiliares no conforman una clase homogénea, sino que debemos distinguir entre auxiliares léxicos y auxiliares funcionales. Luego, hemos refinado las propiedades sintácticas de ambas clases, de manera tal que los auxiliares léxicos son núcleos alrededor de los cuales se configuran «proyecciones extendidas»: cuando una cadena de auxiliares se desplaza a la periferia izquierda, lo hace no monotónicamente (núcleo por núcleo) ni como una unidad (la cadena completa), sino por PPEE, que se adjuntan (en el sentido de Joshi 1985, y trabajos relacionados). La estructura interna de las PPEE nos permite dar cuenta de la imposibilidad de tener al sujeto por encima de la cadena antepuesta, así como la aceptabilidad degradada de posiciones internas a la cadena de auxiliares, cuando el sujeto aparece como dependiente de un auxiliar funcional. 


\section{BIBLIOGRAFÍA}

Abney, S. P. (1987): The English Noun Phrase in its Sentental Aspect. Tesis doctoral. Cambridge: MIT.

Barbosa, P. (2001): «On Inversion in Wh-questions in Romance», en C. Aafke \& J. Hulk \& J.-Y. Pollock (eds.): Subject inversion in Romance and the theory of universal grammar. Oxford: Oxford University Press, pp. 20-59.

BleAm, T. (2000): «Clitic climbing and the power of Tree Adjoining Grammar», en A. Abeille \& O. Rambow (eds.): Tree Adjoining Grammars: Formalisms, linguistic analysis, and processing. Stanford: CSLI, pp. 193-220.

Bravo, A. \& L. García Fernández \& D. Krivochen (2015): «On Auxiliary Chains: Auxiliaries at the Syntax-Semantics Interface», Borealis 4(2), pp. 71-101. https:// doi.org/10.7557/1.4.2.3612

Campos, H. \& M. Zampini (1990): «Focalization strategies in Spanish», Probus 2(1), pp. 47-64. https://doi.org/10.1515/prbs.1990.2.1.47

Chomsky, N. (1957): Syntactic Structures. La Haya: Mouton.

Chomsky, N. (1977): «On Wh-movement», en P. Culicover \& A. Akmajian (eds.): Formal Syntax. New York: Academic Press, pp. 71-133.

Chomsky, N. (1986): Barriers. Cambridge, Mass.: MIT Press.

Chomsky, N. (1995): The Minimalist Program. Cambridge, Mass.: MIT Press.

Chomsky, N. (2001): «Derivation by Phase», en M. Kenstowicz (ed.): Ken Hale: A Life in Language. Cambridge, Mass.: MIT Press, pp. 1-52.

Chomsky, N. (2009): «Opening Remarks», en M. Piattelli-Palmarini \& J. Uriagereka \& P. Salaburu (eds.): Of Minds and Language. Oxford: OUP, pp. 13-43.

Cinque, G. (1999): Adverbs and Functional Heads: A Cross-Linguistic Perspective. Oxford: OUP.

Ciтко, B. (2014): «Multidominance», en C. Boeckx (ed.): The Oxford Handbook of Linguistic Minimalism. Oxford: OUP, pp. 119-142. https://doi.org/10.1093/ oxfordhb/9780199549368.013.0006

De VRIES, M. (2009): «On Multidominance and Linearization», Biolinguistics 3(4), pp. 344-403.

EMONDS, J. (1970): Root and structure preserving transformations. Tesis doctoral. Cambridge: MIT.

EMONDS, J. (2007): «Unspecified categories as the key to root constructions», en J. Emonds: Discovering Syntax: Clause Structures of English, German and Romance. Berlin: Mouton de Gruyter, pp. 331-380. 
Epstein, S. D. \& T. D. Seely (2006): Derivations in minimalism. Cambridge: CUP. https://doi.org/10.1017/CBO9780511550607

ESCANDELl VidAL, M. V. \& M. LeONETti (2009): «La expresión del verum focus en español», Español actual 92, pp. 11-46.

FILlmore, C. (1963): «The Position of Embedding Transformations in a Grammar», Word 19(2), pp. 208-231. https://doi.org/10.1080/00437956.1963.11659796

Frank, R. (1992): Syntactic Locality and Tree Adjoining Grammar: Grammatical, Acquisition and Processing Perspectives. Tesis doctoral. Philadelphia: University of Pennsylvania.

Frank, R. (2002): Phrase Structure Composition and Syntactic Dependencies. Cambridge, Mass.: MIT Press. https://doi.org/10.7551/mitpress/5366.001.0001

García Fernández, L. \& D. G. Krivochen \& A. Bravo (2017): «Aspectos de la semántica y sintaxis de las cadenas de verbos auxiliares en español», Moenia 23, pp. 1-28.

Gómez Torrego, L. (1999): «Los verbos auxiliares. Las perífrasis verbales de infinitivo», en I. Bosque \& V. Demonte (dirs.): Gramática descriptiva de la lengua española. Madrid: Espasa, vol. II, pp. 3323-3389.

Grimshaw, J. (2005): Words and Structure. Stanford: CLSI, pp. 1-74.

GuÉron, J. \& T. Hoekstra (1988): «T-Chains and the Constituent Structure of Auxiliaries», en A. Cardinaletti \& G. Cinque \& G. Giusti (eds.): Constituent Structure. Papers from the 1987 GLOW Conference. Venice: Annali di Ca' Foscari, pp. 35-99.

Hernanz, M. Ll. \& J. M. ${ }^{a}$ Brucart (1987): La Sintaxis. Barcelona: Crítica.

Hopcroft, J. \& J. Ullman (1969): Formal Languages and their relation to Automata. London: Addison-Wesley.

JimÉnEZ FERnÁNDEZ, A. (2015): «Towards a typology of focus: Subject position and microvariation at the discourse-syntax interface», Ampersand 2, pp. 49-60. https://doi.org/10.1016/j.amper.2015.03.001

JimÉNEZ FERNÁNDEZ, A. (en prensa): «Negative Preposing. Intervention and Parametric Variation in Complement Clauses», Atlantis. Journal of the Spanish Association of Anglo-American Studies 39.

Johnson, K. (2016): Towards a Multidominant theory of movement. Ms. Amherst: University of Massachusetts. http://people.umass.edu/kbj/homepage/Content/ Multi_Movement.pdf

Joshi, A. K. (1985): «Tree adjoining grammars: How much context-sensitivity is required to provide reasonable structural descriptions?», en D. Dowty \& L. Karttunen \& A. Zwicky (eds.): Natural Language Parsing. Cambridge, Mass.: CUP, pp. 206-250. https://doi.org/10.1017/CBO9780511597855.007 
Joshi, A. K., \& A. Кroch (1985): Linguistic significance of TAG's. Ms. Disponible en https://www.ling.upenn.edu/ kroch/

Karttunen, L. \& M. KaY (1985): «Structure sharing with binary trees», en Proceedings of the 23rd Association for Computational Linguistics. Chicago, Illinois: University of Chicago, pp. 133-136. https://doi.org/10.3115/981210.981226

KaYne, R. (1994): The Antisymmetry of Syntax. Cambridge, Mass.: MIT Press.

KIPARSKY, P. (1968): «Linguistic universals and linguistic change», en E. Bach \& R. T. Harms (eds.): Universals in linguistic theory. New York: Holt, Rinehart \& Winston, pp. 171-202.

Koutsoudas, A. \& G. Sanders (1979): «On the universality of rules and rule ordering constraints», Studia Linguistica 33(1), pp. 57-78. https://doi. org/10.1111/j.1467-9582.1979.tb00676.x

Koutsoudas, A. \& G. SANDERS \& C. Noll (1974): «The application of phonological rules», Language 50, pp. 1-28. https://doi.org/10.2307/412007

Krivochen, D. (2015): «On Phrase Structure Building and Labeling Algorithms: Towards a Non-Uniform Theory of Syntactic Structures», The Linguistic Review 32(3), pp. 515-572. https://doi.org/10.1515/tlr-2014-0030

Krivochen, D. (2018): Syntax as Graph Theory. Ms. Reading: University of Reading. http://ling.auf.net/lingbuzz/003842

Krivochen, D. \& L. García Fernández (en prensa): «Variability in syntactic-semantic cycles: evidence from auxiliary chains», en M. González Rivera \& S. Sessarego (eds.): At the interface: Essays in honor of Javier Gutiérrez-Rexach. Oxford: Oxford University Press.

Ladusaw, W. (1980): Polarity sensitivity as inherent scope relations. New York: Garland.

LASNIK, H. \& M. SAITO (1984): «On the nature of proper government», Linguistic Inquiry 15, pp. 235-289.

LasniK, H. \& M. Saito (1992): Move Alpha: Conditions on its Application and Output. Cambridge, Mass.: MIT Press.

Leonetti, M. \& M. V. EsCandell Vidal (2009): «Fronting and verum focus in Spanish», en A. Dufter \& D. Jacob (eds.): Focus and Background in Romance Languages. Amsterdam: John Benjamins, pp. 155-204. https://doi.org/10.1075/ slcs.112.07leo

Levine, R. (1985): «Right Node (Non-)Raising», Linguistic Inquiry 16(3), pp. 492-497.

MAY, R. (1985): Logical form: its structure and derivation. Cambridge, Mass.: MIT Press. 
MCCAWLEY, J. D. (1968): «Concerning the base component of a transformational grammar», Foundations of Language 4, pp. 243-269.

McCAWley, J. D. (1982): «Parentheticals and Discontinuous Constituent Structure», Linguistic Inquiry 13(1), pp. 91-106.

McCawley, J. D. (1988): The Syntactic Phenomena of English. Chicago: University of Chicago Press, 2 vols.

OSBORNE, T. (2008): «Major constituents: and two dependency grammar constraints on sharing in coordination», Linguistics 46(6), pp. 1109-1165. https://doi.org/10.1515/ LING.2008.036

Osborne, T. \& M. Putnam \& T. Groß (2011): «Bare phrase structure, label-less trees, and specifier-less syntax. Is Minimalism becoming a dependency grammar?», The Linguistic Review 28(3), pp. 315-364. https://doi.org/10.1515/tlir.2011.009

Pullum, G. K. \& B. C. Scholz (2001): «On the distinction between model-theoretic and generative-enumerative syntactic frameworks», en P. de Groote \& G. Morrill \& C. Retoré (eds.): Logical Aspects of Computational Linguistics: 4th International Conference (Lecture Notes in Artificial Intelligence, 2099). Berlin: Springer Verlag, pp. 17-43. https://doi.org/10.1007/3-540-48199-0_2

RAE-ASALE (= Real Academia Española / Asociación de Academias de la Lengua Española) (2009): Nueva gramática de la lengua española. Madrid: Espasa.

Ringen, C. (1974): «On Arguments for Rule Ordering», Foundations of Language 8(2), pp. 266-273.

Rizzi, L. (2010): «On Some Properties of Criterial Freezing», en P. Panagiotidis (ed.): The Complementizer Phase: Subjects and Operators. Oxford: OUP, pp. 17-32. https://doi.org/10.1093/acprof:oso/9780199584352.003.0002

SAmpson, G. (1975): «The Single Mother Condition», Journal of Linguistics 11(1), pp. 1-11. https://doi.org/10.1017/S0022226700004242

Schmerling, S. F. (1983): «A new theory of English auxiliaries», en F. Heny \& B. Richards (eds.): Linguistic categories: auxiliaries and related puzzles. Dordrecht: Reidel, vol. II, pp. 1-53.

SHIEBER, S. (1986): An Introduction to Unification-Based Approaches to Grammar. Brookline, Mass.: Microtome Publishing.

SLOAN, K. (1991): «Quantifier-Wh Interaction», en L. Lai-Shen Cheng \& H. Demirdache (eds.): More Papers on Wh-movement, MITWPL \#15, pp. 219-237.

Torrego, E. (1984): «On Inversion in Spanish and Some of Its Effects», Linguistic Inquiry 15(1), pp. 103-129. 
UriagereKa, J. (2002): «Multiple Spell-Out», en J. Uriagereka: Derivations: Exploring the Dynamics of Syntax. London: Routledge, pp. 45-65.

URIBE-ETXEBARRIA, M. (1992): «On the structural positions of the subject in Spanish, their nature and their consequences for quantification», en J. Ortiz de Urbina (ed.): Syntactic theory and Basque syntax. San Sebastián: Gipuzkoako Foru Aldundia, pp. 447-491.

ZaGona, K. (2003): The Syntax of Spanish. Cambridge: CUP.

Zubizarreta, L. (2001): «The Constraint on Preverbal Subjects in Romance Interrogatives: A Minimality Effect», en A. C. J. Hulk \& J.-Y. Pollock (eds.): Subject inversion in Romance and the theory of universal grammar. Oxford: Oxford University Press, pp. 183-204.

Zwicky, A. \& S. IsARD (1963): «Some aspects of tree theory», Working Paper W-6674, Bedford, Mass: The MITRE Corporation. Disponible en: https://web.stanford. edu/ Zwicky/some-aspects-of-tree-theory.pdf 